\title{
Spectral geometry of the Moyal plane with harmonic propagation
}

\author{
Victor Gayral and Raimar Wulkenhaar
}

\begin{abstract}
We construct a 'non-unital spectral triple of finite volume' out of the Moyal product and a differential square root of the harmonic oscillator Hamiltonian. We find that the spectral dimension of this triple is $d$ but the KO-dimension is $2 d$. We add another Connes-Lott copy and compute the spectral action of the corresponding Yang-Mills-Higgs model. As result, the 'covariant coordinate' involving the gauge field combines with the Higgs field to a unified potential, yielding a deep unification of discrete and continuous parts of the geometry.
\end{abstract}

Mathematics Subject Classification (2010). 46L87, 81T75.

Keywords. Noncommutative differential geometry, quantum theory: noncommutative geometry methods, non-unital spectral triples, Moyal deformation, heat kernel techniques.

\section{Contents}

1 Introduction . . . . . . . . . . . . . . . . . . . . . . . . . 939

2 The harmonic oscillator spectral triple for Moyal space . . . . . . . . . . . . . . . 941

3 The spectral action . . . . . . . . . . . . . . . . . . . . . . . 961

Appendix: Locally compact noncommutative spin manifolds . . . . . . . . . . . . 975

References . . . . . . . . . . . . . . . . . . . . . . . . 977

\section{Introduction}

Unlike the compact (unital) case [9] and until now, there is for complete non-compact Riemannian spin manifolds no proper reconstruction theorem from a spectral point of view. Thus the question of the defining 'axioms' for non-unital spectral triples is not yet fully answered. However, the basic and most important ideas of modifications for the locally compact case are clear and appeared already in Connes' founding paper [7]. The case of the ordinary Dirac operator of a locally compact complete Riemannian spin manifold manifests that one cannot assume the resolvent of the Dirac type operator underlying a locally compact (non-unital) spectral triple to be a compact operator. The natural replacement is to ask that the 'localized resolvent', i.e., the resolvent multiplied with an element of the algebra, is a compact operator. 
Another issue, explained in depth in [18], is the choice of a unitization of the algebra. This choice is constrained by the orientability condition, which in the unital case (and with an integral metric dimension) is the question of the existence of an Hochschild cycle defining a volume form through the noncommutative integral given by the Dixmier trace. Again, commutative but locally compact examples show that this has to be a Hochschild cycle on a specific unitization of the algebra we start with, but not on the algebra itself. With these two main modifications (compactness of the localized resolvent and existence of a preferred unitization), most of the conditions for a non-unital spectral triple are easy to spell out. Only the Poincaré duality remains unclear to formulate. To help the reader with this discussion, in the appendix we have reproduced the modified conditions for non-unital spectral triples, as in given in [18] with the only modification that the metric and KO-dimensions do not have to coincide, according to the recent formulation of the standard model [8] and the Podleś quantum sphere [12]. Note that these conditions are not far away from those given in [31]. However, in [18], [31] there is an extra assumption of existence of a system of local (or quasi-local) units, akin to the local structure of a non-compact manifold. These locality assumptions have been fully removed in a more recent joint work of one of us [3]. However, in that work, the focus is on the index theoretical side of the notion of a spectral triple, not on the noncommutative generalization of a spin manifold. The definition for a non-unital spectral triple given in [3] is the minimal one, ensuring a well-posed Fredholm index problem with a numerical index computable by means of a local representative of the Chern character in cyclic cohomology.

The present article is devoted to the study of a situation somehow in between the compact (unital) and non-compact (non-unital) setting. Indeed, our Dirac-type operator has compact resolvent alone, but it does not reflect the metric dimension. It is only the localized resolvent which exhibits the correct metric dimension. We term this weird situation as 'non-unital spectral triple of finite volume'. This has, at least, one very nice feature, namely that the spectral action can be defined and computed in the usual way. The main motivation for this example comes from noncommutative quantum field theory.

Because of easy computability, quantum field theory on the Moyal plane is the most-studied toy model for noncommutative quantum field theories. The ultraviolet/infra-red mixing problems arising in these models have been solved by one of us in [23], [24] by the introduction of a modified propagator associated with the harmonic oscillator Hamiltonian. See also [34], [28], [29] for different renormalization proofs. From a physics point of view, the most fascinating property of this model is the behavior of its $\beta$-function [22], [25], [15], [14], which makes it a candidate for non-perturbatively renormalizable quantum field theory in dimension four [30], [33], [26]. We recommend [32] for review and introduction to the literature.

In [27], one of us has sketched a possible spectral triple for Moyal space with harmonic oscillator potential. However, it became clear very soon that working out the mathematical details is a non-trivial issue so that the simpler commutative case was studied first [36]. In this paper, we achieve the construction of a spectral 
triple for a suitable algebra of functions on $\mathbb{R}^{d}$ endowed with the Moyal product, together with a Dirac operator which is a square root of the $d$-dimensional harmonic oscillator Hamiltonian. Few remarks are in order. Firstly, in the same way as to find a differential (and not pseudo-differential) square root of the ordinary Laplacian on $\mathbb{R}^{d}$ where one has to go $2^{\left\lfloor\frac{d}{2}\right\rfloor} \times 2^{\left\lfloor\frac{d}{2}\right\rfloor}$ matrices, to find a differential square root of the $d$-dimensional harmonic oscillator Hamiltonian one has to go $2^{d} \times 2^{d}$ matrices this is the main observation in [27]. The second important remark has to do with the choice of the function algebra with Moyal product. Indeed, there are many non-unital Fréchet algebras of functions with Moyal product that one may use while respecting most of the non-unital spectral triple conditions. But there is only one for which the finiteness axiom is satisfied for a Dirac-type operator given by a square root of the harmonic oscillator Hamiltonian, namely the algebra of Schwartz functions $S\left(\mathbb{R}^{d}\right)$. A similar phenomenon appeared in [18] where it has been shown that with the ordinary Dirac operator of $\mathbb{R}^{d}$, there is only one choice of algebra of functions with Moyal product for which the finiteness axiom is satisfied, namely the $L^{2}$-Sobolev space $W^{2, \infty}\left(\mathbb{R}^{d}\right)$. Lastly, the construction of a Hochschild cycle satisfying the orientability axiom requires (see also [36]) two different differential square roots of the harmonic oscillator Hamiltonian, not only one.

The paper is organized as follows. In Section 2, we introduce two spectral triples with common algebra $\mathcal{A}_{\star}$ given by the set of Schwartz functions $S\left(\mathbb{R}^{d}\right)$ with Moyal product, and two different differential square roots of the harmonic oscillator Hamiltonian acting densely on $\mathscr{H}:=L^{2}\left(\mathbb{R}^{d}\right) \otimes \mathbb{C}^{2^{d}}$. The rest of the section is then devoted to prove that these spectral triples are regular, that the metric dimension is $d$, the KOdimension is $2 d$ and that the dimension spectrum $\mathrm{Sd}$ is $d-\mathbb{N}$. In Section 3, we specialize to the case $d=4$ and after having proven a heat-kernel expansion result adapted to our particular situation, we explicitly compute the spectral action for a Higgs model.

\section{The harmonic oscillator spectral triple for Moyal space}

We consider two Moyal-type deformations $\left(\mathscr{A}_{\star}, \mathscr{D}_{\bullet}, \mathscr{H}\right), \bullet=1,2$, of the (commutative) $d$-dimensional harmonic oscillator spectral triple introduced in [36]. In order to implement the Moyal product, the dimension $d$ must be even.

2.1. An isospectral deformation. On $L^{2}\left(\mathbb{R}^{d}\right)$, we introduce the (unbounded) bosonic creation and annihilation operators

$$
a_{\mu}:=\partial_{\mu}+\widetilde{\Omega} x_{\mu}, \quad a_{\mu}^{*}:=-\partial_{\mu}+\widetilde{\Omega} x_{\mu}, \quad \mu=1, \ldots, d,
$$

satisfying the commutation relations $\left[a_{\mu}, a_{\nu}\right]=\left[a_{\mu}^{*}, a_{v}^{*}\right]=0$ and $\left[a_{\mu}, a_{\nu}^{*}\right]=2 \widetilde{\Omega} \delta_{\mu \nu}$. Here, $\tilde{\Omega}>0$ is a frequency parameter. It is the same as $\omega$ in [36], related to $\Omega$ in [24], [27] by $\widetilde{\Omega}=\frac{2 \Omega}{\theta}$ for a special choice of the Moyal deformation matrix, but preferred 
in the general case. On the exterior algebra $\bigwedge\left(\mathbb{C}^{d}\right)$, we introduce fermionic partners $b_{\mu}, b_{\mu}^{*}$ which fulfill the anticommutation relations $\left\{b_{\mu}, b_{\nu}\right\}=\left\{b_{\mu}^{*}, b_{\nu}^{*}\right\}=0$ and $\left\{b_{\mu}, b_{v}^{*}\right\}=\delta_{\mu \nu}$. Then, on the Hilbert space

$$
\mathscr{H}:=L^{2}\left(\mathbb{R}^{d}\right) \otimes \bigwedge\left(\mathbb{C}^{d}\right) \simeq L^{2}\left(\mathbb{R}^{d}\right) \otimes \mathbb{C}^{2^{d}},
$$

these operators give rise to two selfadjoint operators

$$
\mathscr{D}_{1}:=\mathfrak{Q}_{1}+\mathfrak{Q}_{1}^{*}, \quad \mathscr{D}_{2}:=i \mathfrak{Q}_{2}-i \mathfrak{Q}_{2}^{*},
$$

constructed out of the nilpotent supercharges

$$
\mathfrak{Q}_{1}:=a_{\mu} \otimes b^{\mu *}, \quad \mathfrak{Q}_{1}^{*}:=a_{\mu}^{*} \otimes b^{\mu}, \quad \mathfrak{Q}_{2}:=a_{\mu} \otimes b^{\mu}, \quad \mathfrak{Q}_{2}^{*}:=a_{\mu}^{*} \otimes b^{\mu *},
$$

where Einstein's summation convention is used. Indices are raised or lowered by the Euclidean metric $\delta^{\mu \nu}$ or $\delta_{\mu \nu}$, respectively. The (anti-)commutation relations imply for $\bullet \in\{1,2\}$

$$
\begin{gathered}
\mathscr{D}_{\bullet}^{2}=H \otimes 1-(-1)^{\bullet} \widetilde{\Omega} \otimes \Sigma, \\
H:=\frac{1}{2}\left\{a^{\mu}, a_{\mu}^{*}\right\}=-\partial_{\mu} \partial^{\mu}+\widetilde{\Omega}^{2} x_{\mu} x^{\mu}, \quad \Sigma:=\left[b_{\mu}^{*}, b^{\mu}\right] .
\end{gathered}
$$

We identify $H$ as the Hamiltonian of the $d$-dimensional harmonic oscillator with frequency $\widetilde{\Omega}$. Its spectrum is $\left\{\lambda_{n}=\widetilde{\Omega}\left(\frac{d}{2}+n\right), n \in \mathbb{N}\right\}$, where the eigenvalue $\lambda_{n}$ appears with multiplicity $\left(\begin{array}{c}n+d-1 \\ d-1\end{array}\right)$. Then $\operatorname{Tr}\left(H^{-(d+\varepsilon)}\right) \leq \frac{\widetilde{\Omega}^{-(d+\varepsilon)}}{(d-1) !} \sum_{n=0}^{\infty}(n+$ $\left.\frac{d}{2}\right)^{-(1+\varepsilon)}<\infty$, and from the boundeness of $\Sigma$, it follows that $\left(\left|\mathscr{D}_{\bullet}\right|+1\right)^{-z}, \bullet=1,2$, is trace-class for $\operatorname{Re}(z)>2 d$.

Remark 1. Our choice of $\mathscr{D}_{2}$ differs from [36]. One should take $\mathscr{D}_{2}$ from (1) also for the commutative case to view our spectral triple as isospectral deformation. As seen in the next, the choice (1) is required by the orientability axiom. The commutative version is somehow degenerate and does not detect the sign of the frequency in (2).

We now wish to implement the Moyal product $\star$ in this picture:

$$
f \star g(x)=\int_{\mathbb{R}^{d} \times \mathbb{R}^{d}} \frac{d y d k}{(2 \pi)^{d}} f\left(x+\frac{1}{2} \Theta \cdot k\right) g(x+y) \mathrm{e}^{i\langle k, y\rangle},
$$

parametrized by an invertible skew-symmetric matrix $\Theta^{t}=-\Theta \in M_{d}(\mathbb{R})$. We first need to find out which algebra $\mathcal{A}_{\star}$ of functions (or distributions) with Moyal product to use. For that aim, observe that the finiteness condition alone dictates the choice of the topological vector space underlying the algebra $\mathcal{A}_{\star}$. Indeed, from (2) we conclude for $\bullet=1,2$ that

$$
\mathscr{H}_{\infty}:=\bigcap_{m \geq 0} \operatorname{dom}\left(\mathscr{D}_{\bullet}^{n}\right)=S\left(\mathbb{R}^{d}\right) \otimes \bigwedge\left(\mathbb{C}^{d}\right) \simeq \delta\left(\mathbb{R}^{d}, \mathbb{C}^{2^{d}}\right),
$$

which is required to be a finitely generated projective module over the algebra of the spectral triple. Thus this naturally leads us to the choice $\mathcal{A}_{\star}:=\left(S\left(\mathbb{R}^{d}\right), \star\right)$, with even $d$. 
Remark 2. For the ordinary Dirac operator on the trivial spin bundle of $\mathbb{R}^{d}$, the set of smooth spinors is isomorphic to $W^{2, \infty}\left(\mathbb{R}^{d}\right) \otimes \mathbb{C}^{2^{\left\lfloor\frac{d}{2}\right\rfloor}}$. In this case, the topological vector space underlying the choice of the algebra is the $L^{2}$-Sobolev space $W^{2, \infty}\left(\mathbb{R}^{d}\right)$. Note that the latter is stable under the Moyal product too, and that this is the choice made in [18].

Here are the main properties of the Moyal product we will use later on (for more information see [18]). First is strong closedness

$$
\int f \star g(x) d x=\int f(x) g(x) d x=\int g \star f(x) d x \text { for all } f, g \in L^{2}\left(\mathbb{R}^{d}\right) .
$$

Then we have the Leibniz rule

$$
\partial_{\mu}(f \star g)=\partial_{\mu} f \star g+f \star \partial_{\mu} g
$$

and the following identities

$\left\{f, x^{\mu}\right\}_{\star}:=x^{\mu} \star f+f \star x^{\mu}=2 x^{\mu} f, \quad\left[x^{\mu}, f\right]_{\star}:=x^{\mu} \star f-f \star x^{\mu}=i \Theta^{\mu \nu} \partial_{\nu} f$,

both holding for $f, g \in \mathcal{A}_{\star}$. Finally, there is the (non-unique) factorization property [20], p. 877:

$$
\text { for all } f \in \mathcal{A}_{\star} \text { there exist } g, h \in \mathcal{A}_{\star} \text { such that } f=g \star h .
$$

Following [18], we then specify the preferred unitization $\mathcal{B}_{\star}$ of $\mathcal{A}_{\star}$, as the space of smooth bounded functions on $\mathbb{R}^{d}$ with all partial derivatives bounded. The Moyal product (3) extends to $\mathscr{B}_{\star}$, and $\mathcal{A}_{\star} \subset \mathscr{B}_{\star}$ is an essential two-sided ideal, [18], Theorem 2.21, but is not dense. The reason why we chose this particular unitization is that $\mathscr{B}_{\star}$ contains the plane waves and constant functions (but no other non-constant polynomials) and this is crucial for the orientability condition (see Section 2.3). Note that the $C^{*}$-completion of $\mathscr{B}_{\star}$ is

$$
A_{\star}:=\left\{T \in \mathcal{S}^{\prime}\left(\mathbb{R}^{d}\right) \mid T \star f \in L^{2}\left(\mathbb{R}^{d}\right) \text { for all } f \in L^{2}\left(\mathbb{R}^{d}\right)\right\} .
$$

Indeed, according to [18], Theorem 2.21, $A_{\star}$ coincides via the Weyl quantization map, with the von Neumann algebra of all bounded operators on $L^{2}\left(\mathbb{R}^{\frac{d}{2}}\right)$. Moreover, by the Calderón-Vaillancourt Theorem [2], $\mathscr{B}_{\star}$ is contained (still via the Weyl map) in $A_{\star}$. But the Beals-Cordes characterization [1], [11] says that $\mathscr{B}_{\star}$ is exactly the set of bounded operators which are smooth for the irreducible Schrödinger representation of the Heisenberg group. We conclude using a result of Dixmier and Maillavin [16], which in this context says that the set of smooth operators for the Schrödinger representation is dense in $A_{\star}$.

Therefore, $A_{\star}$ acts on $\mathscr{H}$ by componentwise left Moyal multiplication, that we denote by $L_{\star}$ :

$$
L_{\star}: A_{\star} \times \mathscr{H} \rightarrow \mathcal{H}, \quad(f, \psi \otimes m) \mapsto(f \star \psi) \otimes m,
$$


for $\psi \in L^{2}\left(\mathbb{R}^{d}\right)$ and $m \in \bigwedge\left(\mathbb{C}^{d}\right)$. In particular, we have the bounds [18]:

$$
\begin{aligned}
\left\|L_{\star}(f)\right\| & \leq C(\Theta)\|f\|_{2}, \quad f \in \mathcal{A}_{\star}, \\
\left\|L_{\star}(f)\right\| & \leq C^{\prime}(\Theta) \sup _{|\alpha| \leq d+1}\left\|\partial^{\alpha} f\right\|_{\infty}, \quad f \in \mathcal{B}_{\star} .
\end{aligned}
$$

We also define the (anti-)action $R_{\star}$ of $A_{\star}$ on $\mathscr{H}$ by componentwise right Moyal multiplication:

$$
R_{\star}: \mathcal{A}_{\star} \times \mathscr{H} \rightarrow \mathscr{H}, \quad(f, \psi \otimes m) \mapsto(\psi \star f) \otimes m .
$$

Since the complex conjugation is an involution of the algebra $A_{\star}$, and from the traciality of the Moyal product (4), we get $L_{\star}(f)^{*}=L_{\star}(\bar{f}), R_{\star}(f)^{*}=R_{\star}(\bar{f})$. Moreover, this also shows that the two representations $L_{\star}$ and $R_{\star}$ have the same norms:

$$
\left\|L_{\star}(f)\right\|=\left\|R_{\star}(f)\right\| \text { for all } f \in A_{\star} .
$$

To avoid too many notations, $L_{\star}, R_{\star}$ will also denote the left and right actions of $\mathcal{A}_{\star}$ and $B_{\star}$ on $L^{2}\left(\mathbb{R}^{d}\right)$.

We now check that our spectral triple $\left(\mathcal{A}_{\star}, \mathcal{H}, \mathscr{D}_{\bullet}\right), \bullet=1,2$, defines a non-unital spectral triple with spectral dimension $d$ and KO-dimension $2 d$, in the sense of Definition 25 in the Appendix.

2.2. Boundedness and compactness. From (6), we obtain for $f \in \mathscr{B}_{\star}$ on $\operatorname{dom}\left(\mathscr{D}_{\bullet}\right)$ :

$$
\begin{aligned}
& {\left[\mathcal{D}_{1}, L_{\star}(f)\right]=L_{\star}\left(i \partial_{\mu} f\right) \otimes \Gamma^{\mu},} \\
& {\left[\mathcal{D}_{2}, L_{\star}(f)\right]=L_{\star}\left(i \partial_{\mu} f\right) \otimes \Gamma^{\mu+d},}
\end{aligned}
$$

where $\Gamma^{\mu}:=\left(i b^{\mu}-i b^{* \mu}\right)-\frac{1}{2} \widetilde{\Omega} \Theta^{\mu \nu}\left(b_{v}+b_{v}^{*}\right)$ and $\Gamma^{\mu+d}:=\left(b^{\mu}+b^{* \mu}\right)-$

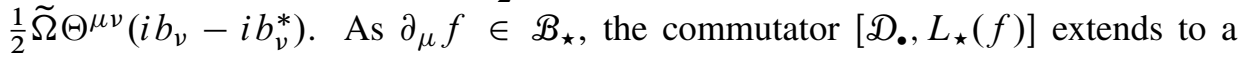
bounded operator. It is a remarkable property of the Moyal algebra that just the $d$-dimensional differential of $f$ appears, no $x$-multiplication.

For the compactness condition, there is not much to say as $\left(\mathscr{D}_{\bullet}+\lambda\right)^{-1}$ is, for $\lambda$ not in the spectrum of $\mathscr{D}_{\bullet}$, already a compact operator on $\mathscr{H}$. Indeed, $\left(\mathscr{D}_{\bullet}+\lambda\right)^{-1}$ is compact if and only if $\left(\mathscr{D}_{\bullet}^{2}+1\right)^{-1}=\left(\left(\mathscr{D}_{\bullet}+i\right)^{-1}\right)^{*}\left(\mathscr{D}_{\bullet}+i\right)^{-1}$ is, and that follows from its spectrum governed by $H$. Then $L_{\star}(f)\left(\mathscr{D}_{\bullet}+\lambda\right)^{-1}$ is compact for any $f \in \mathcal{A}_{\star}$, even for $f \in \mathcal{B}_{\star}$.

2.3. Orientability. Note first that the operators $\Gamma^{\mu}, \Gamma^{\mu+d}$ defined by (9) satisfy the anticommutation relations

$$
\left\{\Gamma^{\mu}, \Gamma^{v}\right\}=\left\{\Gamma^{\mu+d}, \Gamma^{\nu+d}\right\}=2\left(g^{-1}\right)^{\mu \nu}, \quad\left\{\Gamma^{\mu}, \Gamma^{\nu+d}\right\}=0,
$$

where the symmetric matrix $g \in \operatorname{GL}(d, \mathbb{R})$ is defined by

$$
g:=\left(\operatorname{Id}_{d}-\frac{1}{4} \widetilde{\Omega}^{2} \Theta^{2}\right)^{-1}
$$


and plays the role of an effective metric. Note that $\Theta^{2}=-\Theta^{t} \Theta$ is negative definite so that

$$
\left(1+\frac{1}{4} \widetilde{\Omega}^{2}\|\Theta\|^{2}\right)^{-1} \operatorname{Id}_{d} \leq g \leq \operatorname{Id}_{d} .
$$

We will frequently use that $\Theta, g, g^{-1}$ commute with each other. Raising and lowering of summation indices will always be performed with the Euclidean metric $\delta^{\mu \nu}, \delta_{\mu \nu}$.

Thus the $\left\{\Gamma^{1}, \ldots, \Gamma^{2 d}\right\}$ generate a Clifford algebra of double dimension $2 d$. The inverse transformation of (9) reads

$$
i b_{v}-i b_{v}^{*}=g_{v \mu}\left(\Gamma^{\mu}+\frac{1}{2} \widetilde{\Omega} \Theta^{\mu \rho} \Gamma_{\rho+d}\right), \quad b_{v}+b_{v}^{*}=g_{v \mu}\left(\Gamma^{\mu+d}+\frac{1}{2} \widetilde{\Omega} \Theta^{\mu \rho} \Gamma_{\rho}\right) .
$$

Therefore, we can express $\mathscr{D}_{1}$ in terms of $\left\{\Gamma^{1}, \ldots, \Gamma^{2 d}\right\}$, but not in terms of the half set of operators $\left\{\Gamma^{1}, \ldots, \Gamma^{d}\right\}$ produced by the commutator of $\mathscr{D}_{1}$ with $\mathscr{B}_{\star}$. Similar comments apply for $\mathscr{D}_{2}$. In conclusion, we get

$$
\begin{aligned}
& D_{1}=i \partial^{v} \otimes g_{v \mu}\left(\Gamma^{\mu}+\frac{1}{2} \widetilde{\Omega} \Theta^{\mu \rho} \Gamma_{\rho+d}\right)+\widetilde{\Omega} x^{\nu} \otimes g_{v \mu}\left(\Gamma^{\mu+d}+\frac{1}{2} \widetilde{\Omega} \Theta^{\mu \rho} \Gamma_{\rho}\right), \\
& D_{2}=i \partial^{v} \otimes g_{v \mu}\left(\Gamma^{\mu+d}+\frac{1}{2} \widetilde{\Omega} \Theta^{\mu \rho} \Gamma_{\rho}\right)+\widetilde{\Omega} x^{\nu} \otimes g_{v \mu}\left(\Gamma^{\mu}+\frac{1}{2} \widetilde{\Omega} \Theta^{\mu \rho} \Gamma_{\rho+d}\right) .
\end{aligned}
$$

General results for Clifford algebras then show that any element of the Clifford algebra which anticommutes with every $\Gamma^{\mu}$ and $\Gamma^{\mu+d}$ is a multiple of the anti-symmetrized product of the generators $\left\{\Gamma^{1}, \ldots, \Gamma^{2 d}\right\}$. Therefore, a grading operator commutating with $\mathscr{D}_{1}$ cannot be found in the algebra generated by $L_{\star}(f), R_{\star}(f)$ and $\left[\mathscr{D}_{1}, L_{\star}(f)\right]$, so that a (generalized) implementation of the orientability axiom requires both Diractype operators $\mathscr{D}_{1}, \mathcal{D}_{2}$.

Let $u_{\mu}:=e^{-i x_{\mu}} \in \mathcal{B}_{\star}$. We know from [18] that the element

$$
c:=\sum_{\sigma \in S_{d}} \varepsilon(\sigma) \frac{i^{\frac{d(d-1)}{2}} \sqrt{\operatorname{det} g}}{d !}\left(\left(u_{1} \star \ldots \star u_{d}\right)^{-1} \otimes 1\right) \otimes u_{\sigma(1)} \otimes \ldots u_{\sigma(d)}
$$

is a Hochschild $d$-cycle for the algebra $\mathcal{B}_{\star}$, with values in $\mathcal{B}_{\star} \otimes \mathcal{B}_{\star}^{o}$. (In the expression of $c$, the inverse is with respect to the $\star$-product and the scaling by $\sqrt{\operatorname{det} g}$ is irrelevant for cyclicity.) For $\pi_{\mathscr{D}}$. defined in the orientability definition in the Appendix, we then obtain from (9)

$$
\begin{aligned}
& \gamma_{1}:=\pi_{D_{1}}(c)=\frac{\sqrt{\operatorname{det} g}}{d !} i^{\frac{d(d-1)}{2}} \sum_{\sigma \in S_{d}} \varepsilon(\sigma) \otimes \Gamma^{\sigma(1)} \ldots \Gamma^{\sigma(d)}, \\
& \gamma_{2}:=\pi_{D_{2}}(c)=\frac{\sqrt{\operatorname{det} g}}{d !} i^{\frac{d(d-1)}{2}} \sum_{\sigma \in S_{d}} \varepsilon(\sigma) \otimes \Gamma^{\sigma(1)+d} \ldots \Gamma^{\sigma(d)+d},
\end{aligned}
$$

and they satisfy the relations

$$
\gamma_{1}^{2}=1=\gamma_{2}^{2}, \quad \gamma_{1}^{*}=\gamma_{1}, \quad \gamma_{2}^{*}=\gamma_{2}, \quad \gamma_{1} \gamma_{2}=(-1)^{d} \gamma_{2} \gamma_{1} .
$$


The Clifford algebra relations together with (9) then lead to

$$
\begin{aligned}
\operatorname{Tr}_{\wedge\left(\mathbb{C}^{d}\right)}\left(\gamma_{\bullet} L_{\star}\left(f_{0}\right)\left[\mathcal{D}_{\bullet}, L_{\star}\left(f_{1}\right)\right] \ldots\left[\mathcal{D}_{\bullet}, L_{\star}\left(f_{d}\right)\right]\right) \\
\quad=\frac{i^{-\frac{d(d-3)}{2}} 2^{d}}{d ! \sqrt{\operatorname{det} g}} \sum_{\sigma \in S_{d}} \varepsilon(\sigma) L_{\star}\left(f_{0} \star \partial_{\sigma(1)} f_{1} \star \cdots \star \partial_{\sigma(d)} f_{d}\right) .
\end{aligned}
$$

Together with the Dixmier trace formula derived later in Theorem 14 it follows that $c$ satisfies the orientability conditions. Recall that in the unital case, $\pi_{\mathscr{D}}(c)$ is the grading operator which anticommutes with $\mathscr{D}$. As explained above, this is not the case in our example, neither in the commutative example studied in [36]. But we can realize the grading operator in the algebra generated by both spectral triples $\left(\mathcal{A}_{\star}, \mathscr{D}_{1}, \mathscr{H}\right)$ and $\left(\mathcal{A}_{\star}, \mathscr{D}_{2}, \mathscr{H}\right)$ by defining

$$
\Gamma:=(-i)^{d} \gamma_{1} \gamma_{2}=(-i)^{d} \pi_{\mathscr{D}_{1}}(c) \pi_{\mathscr{D}_{2}}(c) .
$$

Since $\boldsymbol{\gamma}_{1}, \boldsymbol{\gamma}_{2}$ commute with every element of $\mathcal{A}_{\star}$ or $\mathscr{B}_{\star}, \Gamma$ does too, and the discussion above shows that

$$
\Gamma^{2}=1, \quad\left\{D_{\bullet}, \Gamma\right\}=0, \quad \bullet=1,2 .
$$

Hence, $\Gamma$ defines the grading operator for the two spectral triples $\left(\mathcal{A}_{\star}, \mathcal{H}, \mathscr{D}_{\bullet}\right), \bullet=$ 1,2 . We stress that the necessity of the two Dirac operators $\mathscr{D}_{1}, \mathscr{D}_{2}$, is quite different from conventional spectral triples [9] where a single operator is needed.

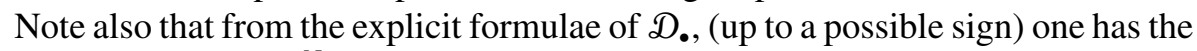
relation $\Gamma=1 \otimes(-1)^{N_{f}}$ in terms of the fermionic number operator $N_{f}=b_{\mu}^{*} b^{\mu}$.

2.4. KO-dimension and other algebraic conditions. The real structure is an antilinear isometry $J$ on $\mathcal{H}$. We assume that for $d$ even the KO-dimension $k$ is even, too. Then according to the sign table in the Appendix we have

$$
J D_{\bullet}=\mathscr{D}_{\bullet} J, \quad \bullet=1,2 .
$$

This is achieved by the following non-trivial action on the matrix part of $\mathscr{H}$,

$$
J a_{\mu} J^{-1}=a_{\mu}, \quad J a_{\mu}^{*} J^{-1}=a_{\mu}^{*}, \quad J b_{\mu} J^{-1}=b_{\mu}^{*}, \quad J b_{\mu}^{*} J^{-1}=b_{\mu},
$$

where we have omitted tensor products with 1 . In particular, conjugation by $J$ preserves the (anti-)commutation relations. We can view $\bigwedge\left(\mathbb{C}^{d}\right)$ as generated by repeated action of $\left\{b_{\mu}^{\dagger}\right\}$ on the vacuum vector $|0\rangle$ defined by $b_{\mu}|0\rangle=0$. It then follows that, up to a prefactor of modulus 1 which cancels in every relation of the dimension table, $J$ is the Hodge- $*$ operator on $\bigwedge\left(\mathbb{C}^{d}\right)$, i.e., is uniquely defined by

$$
J|0\rangle=b_{1}^{*} b_{2}^{*} \ldots b_{d}^{*}|0\rangle,
$$

together with (13) and the anti-linearity $J(z \psi)=\bar{z} J \psi$. In particular, $J \circ L_{\star}(f) \circ$ $J^{-1}=R_{\star}(\bar{f})$, which implements the opposite algebra and achieves the order-one 
condition:

$$
\begin{aligned}
{\left[J L_{\star}\left(f_{1}\right) J^{-1}, L_{\star}\left(f_{2}\right)\right] } & =0, \\
{\left[J L_{\star}\left(f_{1}\right) J^{-1},\left[\mathcal{D}_{\bullet}, L_{\star}\left(f_{2}\right)\right]\right] } & =0 \quad \text { for all } f_{1}, f_{2} \in \mathcal{B}_{\star} .
\end{aligned}
$$

To compute $J^{2}$ we consider, for $\mu_{1}<\mu_{2}<\cdots<\mu_{k}$,

$$
J\left(b_{\mu_{1}}^{*} \ldots b_{\mu_{k}}^{*}|0\rangle\right)=b_{\mu_{1}} \ldots b_{\mu_{k}} b_{1}^{*} b_{2}^{*} \ldots b_{d}^{*}|0\rangle=(-1)^{\sum_{j=1}^{k}\left(\mu_{j}-1\right)} b_{1}^{*} \stackrel{\mu_{1} \ldots \mu_{k}}{\ldots} b_{d}^{*}|0\rangle .
$$

The notation $\stackrel{\mu_{1} \ldots \mu_{k}}{\ldots}$ means that $b_{\mu_{1}}^{*}, \ldots, b_{\mu_{k}}^{*}$ are omitted. We apply $J$ again, to get

$$
\begin{aligned}
J^{2}\left(b_{\mu_{1}}^{*} \ldots b_{\mu_{k}}^{*}|0\rangle\right) & =(-1)^{\sum_{j=1}^{k}\left(\mu_{j}-1\right)} J\left(b_{1}^{*} \stackrel{\mu_{1} \ldots \mu_{k}}{\ldots} b_{d}^{*}|0\rangle\right) \\
& =(-1)^{\sum_{j=1}^{d}(j-1)} b_{\mu_{1}}^{*} \ldots b_{\mu_{k}}^{*}|0\rangle,
\end{aligned}
$$

which means

$$
J^{2}=(-1)^{\frac{d(d-1)}{2}} .
$$

From (9) it follows that $J$ commutes with $\Gamma^{\mu}$ and $\Gamma^{\mu+d}$. From (12) we then conclude $J \Gamma=(-1)^{d} \Gamma J$. Comparing these results with the dimension table in the Appendix, we have proven:

Proposition 3. The spectral geometries $\left(\mathcal{A}_{\star}, \mathscr{H}, \mathcal{D}_{\bullet}, \Gamma, J\right), \bullet=1,2$, for the $d$-dimensional Moyal algebra $\mathcal{A}_{\star}$ are of $\mathrm{KO}$-dimension $2 d \bmod 8$.

2.5. Metric dimension. Since $\mathscr{D}_{\bullet}, \bullet=1,2$, squares (up to matrices) to the $d$-dimensional harmonic oscillator Hamiltonian, we already now that $\left(1+\mathscr{D}_{\bullet}^{2}\right)^{-d}$ belongs to the Dixmier ideal $\mathscr{L}^{1, \infty}(\mathscr{H})$. In this subsection we are going to prove that for the localized operators, the critical dimension is reduced by a factor of 2 , that is for all $f \in \mathcal{A}_{\star}$, the operators $L_{\star}(f)\left(1+\mathscr{D}_{\bullet}^{2}\right)^{-\frac{d}{2}}$ belong to $\mathscr{L}^{1, \infty}(\mathscr{H})$ and that any of its Dixmier traces is a constant multiple of the integral of $f$. To obtain both Dixmier traceability and the value of the Dixmier trace, we will use the results of [4]. In order to do this, we need some preliminary Lemmas (which will also be needed to check the regularity condition, to obtain the dimension spectrum and to compute the spectral action).

Lemma 4. Introducing the operators on $L^{2}\left(\mathbb{R}^{d}\right)$,

$$
\nabla_{\mu}:=\partial_{\mu}+\frac{1}{2} i \tilde{\Omega}^{2} \Theta_{\mu \nu} x^{\nu}, \quad \tilde{\nabla}_{\mu}:=\frac{1}{2}\left(\partial_{\mu}-2 i\left(\Theta^{-1}\right)_{\mu \nu} x^{\nu}\right), \quad \mu=1, \ldots, d,
$$

we have the following relations for $f \in \mathcal{B}_{\star}$ :

$$
\begin{gathered}
{\left[H, L_{\star}(f)\right]=-L_{\star}\left(\left(g^{-1}\right)^{\mu \nu} \partial_{\mu} \partial_{\nu} f\right)-2 L_{\star}\left(\partial^{\mu} f\right) \nabla_{\mu},} \\
{\left[\nabla^{\mu}, L_{\star}(f)\right]=L_{\star}\left(\left(g^{-1}\right)^{\mu \nu} \partial_{\nu} f\right), \quad\left[\tilde{\nabla}^{\mu}, L_{\star}(f)\right]=L_{\star}\left(\partial_{\mu} f\right),} \\
{\left[H, \nabla_{\mu}\right]=-2 i \widetilde{\Omega}^{2} \Theta_{\mu \nu} \tilde{\nabla}^{\nu}, \quad\left[H, \tilde{\nabla}_{\mu}\right]=2 i\left(\Theta^{-1}\right)_{\mu \nu} \nabla^{\nu},} \\
{\left[\tilde{\nabla}_{\mu}, \tilde{\nabla}_{\nu}\right]=i\left(\Theta^{-1}\right)_{\mu \nu}, \quad\left[\nabla_{\mu}, \nabla_{\nu}\right]=-i \widetilde{\Omega}^{2} \Theta_{\mu \nu}, \quad\left[\nabla^{\mu}, \tilde{\nabla}_{\nu}\right]=i\left(g^{-1}\right)^{\mu \rho}\left(\Theta^{-1}\right)_{\rho \nu} .}
\end{gathered}
$$


Proof. This follows from the relations (2), (5) and (6).

Corollary 5. Let $P_{\alpha}(\hat{\nabla})$ be an element of order $\alpha$ of the polynomial algebra generated by $\nabla, \tilde{\nabla}$. Then $P_{\alpha}(\hat{\nabla})(1+H)^{-\alpha / 2}$ extends to a bounded operator.

Proof. From the operator inequalities (no summations on $\mu$ but summation on $v$ )

$$
\left|(1+H)^{-\frac{1}{2}} \partial_{\mu}\right|^{2}=-\partial_{\mu}(1+H)^{-1} \partial_{\mu} \leq-\partial_{\mu}\left(1-\partial_{\nu} \partial^{\nu}\right)^{-1} \partial_{\mu},
$$

and

$$
\left|(1+H)^{-\frac{1}{2}} x_{\mu}\right|^{2}=x_{\mu}(1+H)^{-1} x_{\mu} \leq x_{\mu}\left(1+\widetilde{\Omega}^{2} x_{\nu} x^{\nu}\right)^{-1} x_{\mu},
$$

we see that $\hat{\nabla}_{\mu}(1+H)^{-\frac{1}{2}}$ is bounded. Then the general case follows by induction using

$$
\hat{\nabla}_{\mu_{1}} \hat{\nabla}_{\mu_{2}}(1+H)^{-1}=\hat{\nabla}_{\mu_{1}}(1+H)^{-1} \hat{\nabla}_{\mu_{2}}+\hat{\nabla}_{\mu_{1}}\left[\hat{\nabla}_{\mu_{2}},(1+H)^{-1}\right],
$$

and

$$
\hat{\nabla}_{\mu_{1}}\left[\hat{\nabla}_{\mu_{2}},(1+H)^{-1}\right]=-\hat{\nabla}_{\mu_{1}}(1+H)^{-1}\left[\hat{\nabla}_{\mu_{2}}, H\right](1+H)^{-1},
$$

which is bounded, too, according to Lemma 4.

The following proposition will be crucial for the computation of the spectral action, for the estimate we need to evaluate the Dixmier trace and to compute the dimension spectrum and the residues of the associated zeta functions.

Proposition 6. For $f \in \mathscr{B}_{\star}$, define $\mathcal{T}_{\mu_{1} \ldots \mu_{k}}(f):=\operatorname{Tr}\left(L_{\star}(f) \nabla_{\mu_{1}} \ldots \nabla_{\mu_{k}} e^{-t H}\right)$. Then one has

$$
\begin{aligned}
\mathcal{T}_{\mu_{1}, \ldots \mu_{k}}(f)= & \sum_{1 \leq j_{1}<j_{2}<\cdots<j_{2 a} \leq k}\left(\frac{\tilde{\Omega}}{2 \pi \sinh (2 \widetilde{\Omega} t)}\right)^{\frac{d}{2}} \int_{\mathbb{R}^{d}} d z \sqrt{\operatorname{det} g} f(z) \\
& \cdot e^{-\widetilde{\Omega} \tanh (\tilde{\Omega} t)\langle z, g z\rangle}\left(\mathcal{Z}_{\mu_{1}} \stackrel{j_{1} \ldots j_{2} a}{\cdots} Z_{\mu_{k}}\right)\left(\mathcal{N}_{\mu_{j_{1}} \mu_{j_{2}}} \ldots \mathcal{N}_{\mu_{j_{2 a-1}} \mu_{j_{2} a}}\right),
\end{aligned}
$$

where

$$
\begin{aligned}
\mathcal{Z}_{\mu}:= & -\widetilde{\Omega} \tanh (\tilde{\Omega} t) z_{\mu}+i \widetilde{\Omega}^{2}(\Theta g z)_{\mu}, \\
\mathcal{N}_{\mu \nu}:= & -\frac{1}{2} \widetilde{\Omega}(\operatorname{coth}(\widetilde{\Omega} t)+\tanh (\widetilde{\Omega} t))\left(g^{-1}\right)_{\mu \nu} \\
& -\frac{1}{2} \widetilde{\Omega}^{3} \operatorname{coth}(\widetilde{\Omega} t)(\Theta g \Theta)_{\mu \nu}-\frac{1}{2} i \widetilde{\Omega}^{2} \Theta_{\mu \nu},
\end{aligned}
$$

and $\stackrel{j_{1} \ldots j_{2} a}{\ldots}$ means that $\left\{\mathfrak{Z}_{\mu_{j_{1}}}, \ldots \mathcal{Z}_{\mu_{j_{2 a} a}}\right\}$ are omitted in the product $\mathfrak{Z}_{\mu_{1}} \ldots \mathcal{Z}_{\mu_{k}}$. (Remember that $g$ is a constant metric so that $\sqrt{\operatorname{det} g}$ can also be taken in front of the integral.)

In particular,

$$
\operatorname{Tr}\left(e^{-t H}\right)=(2 \sinh (\widetilde{\Omega} t))^{-d} .
$$


Proof. Since $L_{\star}(f) \nabla_{\mu_{1}} \ldots \nabla_{\mu_{k}} e^{-t H}$ is trace-class (because $\nabla_{\mu_{1}} \ldots \nabla_{\mu_{k}} e^{-t H / 2}$ is bounded by Corollary 5 and $e^{-t H / 2}$ is trace-class), the trace can be evaluated as the integral of the kernel on the diagonal. Thus, in integral kernel representation, we have to compute

$$
\begin{gathered}
\mathcal{T}_{\mu_{1}, \ldots \mu_{k}}(f)=\int_{\mathbb{R}^{d} \times \mathbb{R}^{d}} d x d y\left(L_{\star}(f)\right)(x, y)\left(\frac{\partial}{\partial y^{\mu_{1}}}+\frac{i}{2} \widetilde{\Omega}^{2} \Theta_{\mu_{1} v_{1}} y^{\nu_{1}}\right) \ldots \\
\ldots\left(\frac{\partial}{\partial y^{\mu_{k}}}+\frac{i}{2} \widetilde{\Omega}^{2} \Theta_{\mu_{k} v_{k}} y^{\nu_{k}}\right)\left(e^{-t H}(y, x)\right) .
\end{gathered}
$$

The operator kernel of $e^{-t H}$ is the Mehler kernel

$$
e^{-t H}(x, y)=\left(\frac{\tilde{\Omega}}{2 \pi \sinh (2 \widetilde{\Omega} t)}\right)^{\frac{d}{2}} e^{-\frac{\widetilde{\Omega}}{4} \operatorname{coth}(\widetilde{\Omega} t)\|x-y\|^{2}-\frac{\widetilde{\Omega}}{4} \tanh (\widetilde{\Omega} t)\|x+y\|^{2}},
$$

while the operator kernel of $L_{\star}(f)$ is readily identified to be

$$
L_{\star}(f)(x, y)=\frac{1}{\pi^{d} \operatorname{det} \Theta} \int_{\mathbb{R}^{d}} d z f(z) \mathrm{e}^{i\left\langle x-y, \Theta^{-1}(x+y)\right\rangle+2 i\left\langle z, \Theta^{-1}(x-y)\right\rangle},
$$

where det $\Theta>0$ as square of the Pfaffian of an invertible skew-symmetric matrix. We introduce $u=x-y$ and $v=x+y$ and

$$
\begin{gathered}
\mathfrak{D}_{\mu}(u, v):=\frac{\widetilde{\Omega}}{2} \operatorname{coth}(\widetilde{\Omega} t) u_{\mu}-\frac{\widetilde{\Omega}}{2} \tanh (\tilde{\Omega} t) v_{\mu}+\frac{i}{4} \widetilde{\Omega}^{2} \Theta_{\mu \alpha}\left(v^{\alpha}-u^{\alpha}\right), \\
y_{\mu v}:=-\frac{\tilde{\Omega}}{2}(\operatorname{coth}(\tilde{\Omega} t)+\tanh (\tilde{\Omega} t)) \delta_{\mu \nu}-\frac{i}{2} \widetilde{\Omega}^{2} \Theta_{\mu \nu},
\end{gathered}
$$

to obtain

$$
\begin{aligned}
& \tilde{T}_{\mu_{1}, \ldots \mu_{k}}(f)=\sum_{1 \leq j_{1}<j_{2}<\cdots<j_{2} a \leq k}\left(\frac{\tilde{\Omega}}{2 \pi \sinh (2 \tilde{\Omega} t)}\right)^{\frac{d}{2}} \frac{1}{(2 \pi)^{d} \operatorname{det} \Theta} \int d u d v d z f(z) \\
& \cdot \mathfrak{D}_{\mu_{1}}(u, v) \stackrel{j_{1} \ldots j_{2} a}{\ldots} \mathfrak{D}_{\mu_{k}}(u, v) \mathcal{y}_{\mu_{j_{1}} \mu_{j_{2}}} \ldots \mathcal{Y}_{\mu_{j_{2 a-1}} \mu_{j_{2 a}}} \\
& \cdot e^{-\frac{1}{2}\langle(u, v), Q(u, v)\rangle-\left\langle(u, v),\left(2 i \Theta^{-1} z, 0\right)\right\rangle} \\
& =\sum_{1 \leq j_{1}<j_{2}<\cdots<j_{2 a} \leq k}\left(\frac{\tilde{\Omega}}{2 \pi \sinh (2 \tilde{\Omega} t)}\right)^{\frac{d}{2}} \frac{1}{\operatorname{det} \Theta \sqrt{\operatorname{det} Q}} \int_{\mathbb{R}^{d}} d z f(z) \\
& \left.\mathfrak{D}_{\mu_{1}}\left(\frac{i \partial}{\partial \xi}, \frac{i \partial}{\partial \eta}\right) \stackrel{j_{1} \ldots j_{2} a}{\ldots} \mathfrak{D}_{\mu_{k}}\left(\frac{i \partial}{\partial \xi}, \frac{i \partial}{\partial \eta}\right) \mathcal{Y}_{\mu_{j_{1}} \mu_{j_{2}}} \ldots \mathcal{Y}_{\mu_{j_{2 a-1}} \mu_{j_{2} a}} \mathcal{E}\right|_{\xi=\eta=0}
\end{aligned}
$$

where $\mathcal{E}:=e^{-\frac{1}{2}\left\langle\left(-2 z \Theta^{-1}+\xi, \eta\right), Q^{-1}\left(2 \Theta^{-1} z+\xi, \eta\right)\right\rangle}$ and $Q \in M_{2 d}(\mathbb{C})$ is given by

$$
Q=\left(\begin{array}{cc}
\frac{\tilde{\Omega}}{2} \operatorname{coth}(\tilde{\Omega} t) \operatorname{Id}_{d} & -i \Theta^{-1} \\
i \Theta^{-1} & \frac{\tilde{\Omega}}{2} \tanh (\widetilde{\Omega} t) \operatorname{Id}_{d}
\end{array}\right)
$$


Recalling that $g^{-1}=1-\frac{\widetilde{\Omega}^{2}}{4} \Theta^{2}$ and $g \Theta=\Theta g$, we find by writing $Q$ as product of triangular matrices

$$
\operatorname{det} Q=\frac{1}{\operatorname{det} g(\operatorname{det} \Theta)^{2}}, \quad Q^{-1}=\left(\begin{array}{cc}
-\frac{\tilde{\Omega}}{2} \tanh (\tilde{\Omega} t) g \Theta^{2} & -i g \Theta \\
i g \Theta & -\frac{\tilde{\Omega}}{2} \operatorname{coth}(\tilde{\Omega} t) g \Theta^{2}
\end{array}\right),
$$

so that

$$
\begin{aligned}
& \mathcal{E}=\exp \{-\widetilde{\Omega} \tanh (\widetilde{\Omega} t)\langle z, g z\rangle-\widetilde{\Omega} \tanh (\widetilde{\Omega} t)\langle z, g \Theta \xi\rangle-2 i\langle z, g \eta\rangle \\
& \left.+\frac{\widetilde{\Omega}}{4} \tanh (\widetilde{\Omega} t)\langle\xi, \Theta g \Theta \xi\rangle+i\langle\xi, \Theta g \eta\rangle+\frac{\widetilde{\Omega}}{4} \operatorname{coth}(\widetilde{\Omega} t)\langle\eta, \Theta g \Theta \eta\rangle\right\}, \\
& \mathfrak{D}_{\mu}\left(\frac{i \partial}{\partial \xi}, \frac{i \partial}{\partial \eta}\right) \mathcal{E}=\left(i \widetilde{\Omega}^{2}(\Theta g z)_{\mu}-\widetilde{\Omega} \tanh (\tilde{\Omega} t) z_{\mu}+i \frac{\widetilde{\Omega}^{2}}{2}(\Theta g \Theta \xi)_{\mu}\right. \\
& -\tilde{\Omega} \operatorname{coth}(\tilde{\Omega} t)(g \Theta \eta)_{\mu}+\frac{\tilde{\Omega}}{2} \operatorname{coth}(\tilde{\Omega} t)(\Theta \eta)_{\mu} \\
& \left.-\frac{\tilde{\Omega}}{2} \tanh (\widetilde{\Omega} t)(\Theta \xi)_{\mu}\right) \mathcal{E} \text {. }
\end{aligned}
$$

Then the functions

$$
\begin{aligned}
\mathcal{Z}_{\mu} & :=\left.\mathcal{E}^{-1} \mathfrak{D}_{\mu}\left(\frac{i \partial}{\partial \xi}, \frac{i \partial}{\partial \eta}\right) \mathcal{E}\right|_{\xi=\eta=0}, \\
\mathcal{N}_{\mu \nu} & :=y_{\mu \nu}+\mathfrak{D}_{\mu}\left(\frac{i \partial}{\partial \xi}, \frac{i \partial}{\partial \eta}\right)\left(\mathcal{E}^{-1} \mathfrak{D}_{\nu}\left(\frac{i \partial}{\partial \xi}, \frac{i \partial}{\partial \eta}\right) \mathcal{E}\right),
\end{aligned}
$$

take the form given in the proposition, and the assertion follows.

A very nice feature of the results of [4] is that both the questions of the Dixmier traceability and of the value of the Dixmier trace of an operator of the form $a G^{k}$ are reduced to the value of the Hilbert-Schmidt norm of the heat-type operator $a e^{-t G^{-1}}$. In our context $a=L_{\star}(f), G=\left(1+\mathscr{D}_{\bullet}^{2}\right)^{-1}$, and all we need to do is to evaluate the Hilbert-Schmidt norm of $L_{\star}(f) e^{-t \mathfrak{D}_{\bullet}^{\dot{2}}}$.

Lemma 7. If $f \in \mathcal{A}_{\star}$, then we have

$$
\left\|L_{\star}(f) e^{-t \mathscr{D}_{\bullet}^{2}}\right\|_{2}^{2}=\frac{\widetilde{\Omega}^{\frac{d}{2}}}{\pi^{\frac{d}{2}} \tanh ^{\frac{d}{2}}(2 \tilde{\Omega} t)} \int_{\mathbb{R}^{d}} d z \sqrt{\operatorname{det} g} \bar{f} \star f(z) e^{-\tilde{\Omega} \tanh (2 \tilde{\Omega} t)\langle z, g z\rangle} .
$$

Proof. Since $\mathscr{D}_{\bullet}^{2}=H \otimes 1-(-1)^{\bullet} \tilde{\Omega} \otimes \Sigma$, we have

$$
0 \leq e^{-t D_{\bullet}^{2}}=e^{-t H} \otimes e^{(-1)^{\bullet} t \tilde{\Omega} \Sigma},
$$

and thus

$$
\left\|L_{\star}(f) e^{-t \mathscr{D}_{\bullet}^{2}}\right\|_{2}=\left\|L_{\star}(f) e^{-t H}\right\|_{2} \operatorname{tr}\left(e^{(-1)^{\bullet} 2 t \tilde{\Omega} \Sigma}\right)^{\frac{1}{2}} .
$$

For the matrix trace, we have

$$
\operatorname{tr}\left(e^{(-1)^{\bullet} t \tilde{\Omega} \Sigma}\right)=\operatorname{tr}\left(e^{(-1)^{\bullet} t \tilde{\Omega} \sum_{\mu=1}^{d}\left(b_{\mu}^{*} b_{\mu}-b_{\mu} b_{\mu}^{*}\right)}\right)=\operatorname{tr}\left(\prod_{\mu=1}^{d} e^{(-1)^{\bullet} t \tilde{\Omega}\left(b_{\mu}^{*} b_{\mu}-b_{\mu} b_{\mu}^{*}\right)}\right) .
$$


In the basis $\left|s_{1}, \ldots, s_{d}\right\rangle:=\left(b_{1}^{*}\right)^{s_{1}} \ldots\left(b_{d}^{*}\right)^{s_{d}}|0, \ldots, 0\rangle$ of $\mathbb{C}^{2^{d}}$, with $s_{i} \in\{0,1\}$, we have

$$
-\left(b_{\mu}^{*} b_{\mu}-b_{\mu} b_{\mu}^{*}\right)\left|s_{1}, \ldots, s_{d}\right\rangle=(-1)^{s_{\mu}}\left|s_{1}, \ldots, s_{d}\right\rangle,
$$

and therefore, for both $\bullet=1,2$,

$$
\operatorname{tr}\left(e^{(-1)^{\bullet} t \tilde{\Omega} \Sigma}\right)=2^{d} \cosh ^{d}(\widetilde{\Omega} t) .
$$

The other bit, $\left\|L_{\star}(f) e^{-t H}\right\|_{2}^{2}=\operatorname{Tr}\left(e^{-t H} L_{\star}(\bar{f} \star f) e^{-t H}\right)=\operatorname{Tr}\left(L_{\star}(\bar{f} \star f) e^{-2 t H}\right)$, has been computed in Proposition 6.

Remark 8. Since for $f \in \mathcal{A}_{\star}, \bar{f} \star f$ is a priori not a positive function, in the previous Lemma, one may wonder why $\int d z \sqrt{\operatorname{det} g} \bar{f} \star f(z) \exp \{-\widetilde{\Omega} \tanh (2 \widetilde{\Omega} t)\langle z, g z\rangle\}$ is positive, as it should be. This follows from the following facts: For $A$ a positive definite matrix commuting with $\Theta$, set $g_{A}(x):=e^{-\langle x, A x\rangle}$. Then a computation gives

$$
g_{A} \star g_{A}=\left(\operatorname{det}\left(1+\Theta^{t} A^{2} \Theta\right)\right)^{-\frac{1}{2}} g_{B} \quad \text { with } B=\frac{2 A}{1+\Theta^{t} A^{2} \Theta} .
$$

It follows

$$
\exp \{-\widetilde{\Omega} \tanh (2 \widetilde{\Omega} t)\langle z, g z\rangle\}=\left(\operatorname{det}\left(1+\Theta^{t} A^{2} \Theta\right)\right)^{\frac{1}{2}} g_{A} \star g_{A}
$$

for

$$
A=\frac{g^{-1}-\left(g^{-2}-\widetilde{\Omega}^{2} \tanh (2 \widetilde{\Omega} t)^{2} \Theta^{t} \Theta\right)^{\frac{1}{2}}}{\widetilde{\Omega} \tanh (2 \widetilde{\Omega} t) \Theta^{t} \Theta} .
$$

Note that $g^{-2}-\widetilde{\Omega}^{2} \Theta^{t} \Theta=\left(1-\widetilde{\Omega}^{2} \Theta^{t} \Theta / 4\right)^{2}$ so that $A$ exists for all $t$. Using the traciality of the Moyal product (4), we then get for the matrix $A$ given above and up to a positive constant:

$$
\begin{aligned}
\int d z \bar{f} \star & f(z) \exp \left\{-\tilde{\Omega} \tanh (2 \tilde{\Omega} t)\left\langle z, g^{-1} z\right\rangle\right\} \\
& =C(\Theta, \tilde{\Omega}, t) \int d z \bar{f} \star f(z) g_{A} \star g_{A}(z) \\
& =C(\Theta, \tilde{\Omega}, t) \int d z \bar{f} \star f \star g_{A} \star g_{A}(z) \\
& =C(\Theta, \widetilde{\Omega}, t) \int d z \overline{f \star g_{A}} \star f \star g_{A}(z) \\
& =C(\Theta, \widetilde{\Omega}, t) \int d z \overline{f \star g_{A}}(z) f \star g_{A}(z) \\
& =C(\Theta, \widetilde{\Omega}, t)\left\|f \star g_{A}\right\|_{2} \geq 0 .
\end{aligned}
$$

Moreover, it explains why $L_{\star}(f) e^{-t \mathscr{D}_{\bullet}^{2}}$ is Hilbert-Schmidt also for $f$ in $\mathcal{B}_{\star}$, since $\mathcal{A}_{\star}$ is an ideal of $\mathcal{B}_{\star}$ and $\mathcal{A}_{\star} \subset L^{2}\left(\mathbb{R}^{d}\right)$. 
Lemma 9. For all $t>0$ and $\bullet=1,2$, we have

$$
\operatorname{Tr}\left(e^{-t \mathscr{D}^{2}}\right)=\operatorname{coth}^{d}(\widetilde{\Omega} t) .
$$

Proof. This is a corollary of Lemma 7 and the remark which follows it, by letting $f$ going to the constant unit function.

Lemma 10. If $f \in \mathcal{A}_{\star}$ and $t>0$, then we have the bound

$$
\left\|L_{\star}(f) e^{-t \mathscr{D}_{\bullet}^{2}}\right\|_{2} \leq C\|\bar{f} \star f\|_{1}^{\frac{1}{2}} \max \left(1, t^{-d / 4}\right),
$$

where the constant depends only on $\widetilde{\Omega}$ and $\Theta$.

Proof. This is a direct consequence of Lemma 7.

Lemma 11. There is $C^{\prime}>0$ such that for any $f_{1}, f_{2} \in \mathcal{A}_{\star}$ and $t>0$ one has

$$
\left\|L_{\star}\left(f_{1}\right)\left[L_{\star}\left(f_{2}\right), e^{-t \mathscr{D}_{\bullet}^{2}}\right]\right\|_{1} \leq C^{\prime} t^{\frac{1}{2}} \sum_{\mu=1}^{d}\left\|f_{1}\right\|_{2}\left\|L_{\star}\left(\partial_{\mu} f_{2}\right) e^{-t \mathscr{D}_{\bullet}^{2} / 4}\right\|_{1} .
$$

Proof. By Lemma 9, $e^{-t \mathfrak{D}_{\bullet}^{2}}$ is trace class for $t>0$. We use the identity

$$
\left[e^{A}, B\right]=\int_{0}^{1} d s \frac{d}{d s}\left(e^{s A} B e^{(1-s) A}\right)=\int_{0}^{1} d s e^{s A}[A, B] e^{(1-s) A},
$$

to get

$$
L_{\star}\left(f_{1}\right)\left[L_{\star}\left(f_{2}\right), e^{-t \mathscr{D}_{\bullet}^{2}}\right]=-t L_{\star}\left(f_{1}\right) \int_{0}^{1} d s e^{-t s \mathscr{D}_{\bullet}^{2}}\left[\mathscr{D}_{\bullet}^{2}, L_{\star}\left(f_{2}\right)\right] e^{-t(1-s) \mathscr{D}_{\bullet}^{2}} .
$$

Hence we have

$$
\begin{aligned}
& \left\|L_{\star}\left(f_{1}\right)\left[L_{\star}\left(f_{2}\right), e^{-t \mathscr{D}_{\bullet}^{2}}\right]\right\|_{1} \\
& \leq t\left\|L_{\star}\left(f_{1}\right)\right\| \int_{0}^{1} d s\left(\left\|e^{-t s \mathscr{D}_{\bullet}^{2} / 2} \mathscr{D}_{\bullet}\right\|\left\|e^{-t s \mathscr{D}_{\bullet}^{2} / 2}\left[\mathscr{D}_{\bullet}, L_{\star}\left(f_{2}\right)\right] e^{-t(1-s) \mathscr{D}_{\bullet}^{2}}\right\|_{1}\right. \\
& \left.\quad+\left\|e^{-t s \mathscr{D}_{\bullet}^{2}}\left[\mathscr{D}_{\bullet}, L_{\star}\left(f_{2}\right)\right] e^{-t(1-s) \mathscr{D}_{\bullet}^{2} / 2}\right\|_{1}\left\|\mathscr{D}_{\bullet} e^{-t(1-s) \mathscr{D}_{\bullet}^{2} / 2}\right\|\right) .
\end{aligned}
$$

By spectral theory, $\left\|\mathscr{D}_{\bullet} e^{-t \mathscr{D}_{\bullet}^{2}}\right\|=(2 e t)^{-\frac{1}{2}}$. Thus, using the relation

$$
\left[D_{\bullet}, L_{\star}\left(f_{2}\right)\right]= \begin{cases}i L_{\star}\left(\partial_{\mu} f_{2}\right) \otimes \Gamma^{\mu}, & \bullet=1, \\ i L_{\star}\left(\partial_{\mu} f_{2}\right) \otimes \Gamma^{\mu+4}, & \bullet=2,\end{cases}
$$

we get, with $\left\|L_{\star}\left(f_{1}\right)\right\| \leq C\left\|f_{1}\right\|_{2}$ and $C^{\prime}:=C \sqrt{2}, \pi e^{-\frac{1}{2}} \sup _{\mu=1}^{2 d}\left\|\Gamma^{\mu}\right\|$

$$
\begin{aligned}
& \left\|L_{\star}\left(f_{1}\right)\left[L_{\star}\left(f_{2}\right), e^{-t \mathscr{D}_{\bullet}^{2}}\right]\right\|_{1} \\
& \leq \frac{C^{\prime}}{\pi} t^{\frac{1}{2}}\left\|f_{1}\right\|_{2} \sum_{\mu=1}^{d} \int_{0}^{1} d s s^{-\frac{1}{2}}(1-s)^{-\frac{1}{2}}\left\|e^{-t s D_{\bullet}^{2} / 2} L_{\star}\left(\partial_{\mu} f_{2}\right) e^{-t(1-s) D_{\bullet}^{2} / 2}\right\|_{1} .
\end{aligned}
$$


Estimating

$$
\left\|e^{-t s \mathscr{D}_{\bullet}^{2} / 2} L_{\star}\left(\partial_{\mu} f_{2}\right) e^{-t(1-s) \mathscr{D}_{\bullet}^{2} / 2}\right\|_{1} \leq \begin{cases}\left\|L_{\star}\left(\partial_{\mu} f_{2}\right) e^{-t \mathscr{D}_{\bullet}^{2} / 4}\right\|_{1} & \text { if } s \in\left[0, \frac{1}{2}\right], \\ \left\|e^{-t \mathscr{D}_{\bullet}^{2} / 4} L_{\star}\left(\partial_{\mu} f_{2}\right)\right\|_{1} & \text { if } s \in\left[\frac{1}{2}, 1\right],\end{cases}
$$

the result follows.

Lemma 12. Let $f \in \mathcal{A}_{\star}$. Then there exists a finite constant $C(f)$ such that

$$
\left\|L_{\star}(f) e^{-t \mathscr{D}_{\bullet}^{2}}\right\|_{1} \leq C(f) \max \left(t^{-\frac{d}{2}}, t^{\frac{d}{2}}\right)
$$

for all $t>0$

Proof. Our strategy is to iterate a combination of the factorization property (7) with Lemma 10 and Lemma 11 far enough so that we can bound $e^{-\varepsilon t \mathscr{D}_{\bullet}^{2}}$ alone in trace-norm (i.e., without element of the algebra on both sides).

According to (7), for all $f \in \mathcal{A}_{\star}$ there exist $f_{1}, f_{2} \in \mathcal{A}_{\star}$ such that $f=f_{1} \star f_{2}$, giving

$$
L_{\star}(f) e^{-t \mathscr{D}_{\bullet}^{2}}=L_{\star}\left(f_{1}\right) e^{-t \mathscr{D}_{\bullet}^{2}} L_{\star}\left(f_{2}\right)+L_{\star}\left(f_{1}\right)\left[L_{\star}\left(f_{2}\right), e^{-t \mathscr{D}_{\bullet}^{2}}\right] .
$$

From Lemma 10 and Lemma 11 we conclude

$$
\begin{aligned}
& \left\|L_{\star}(f) e^{-t \mathscr{D}_{\bullet}^{2}}\right\|_{1} \\
& \leq\left\|L_{\star}\left(f_{1}\right) e^{-t \mathscr{D}_{\bullet}^{2} / 2}\right\|_{2}\left\|e^{-t \mathscr{D}_{\bullet}^{2} / 2} L_{\star}\left(f_{2}\right)\right\|_{2}+\left\|L_{\star}\left(f_{1}\right)\left[L_{\star}\left(f_{2}\right), e^{-t \mathscr{D}_{\bullet}^{2}}\right]\right\|_{1} \\
& \leq\left\|\bar{f}_{1} \star f_{1}\right\|_{1}^{\frac{1}{2}}\left\|\bar{f}_{2} \star f_{2}\right\|_{1}^{\frac{1}{2}} \max \left(t^{-\frac{d}{2}}, 1\right) \\
& \quad+C^{\prime} t^{\frac{1}{2}} \sum_{\mu=1}^{d}\left\|f_{1}\right\|_{2}\left\|L_{\star}\left(\partial_{\mu} f_{2}\right) e^{-t D_{\bullet}^{2} / 4}\right\|_{1} .
\end{aligned}
$$

Iterating $d$-times the estimate (16) with the repeated factorization

$$
\partial_{\mu_{1}} f_{2}=f_{1, \mu} \star f_{2, \mu}, \ldots, \partial_{\mu_{k+1}} f_{2, \mu_{1} \ldots \mu_{k}}=f_{1, \mu_{1} \ldots \mu_{k+1}} \star f_{2, \mu_{1} \ldots \mu_{k+1}},
$$

with $f_{1, \mu}, f_{2, \mu}, \ldots, f_{1, \mu_{1} \ldots \mu_{k+1}}, f_{2, \mu_{1} \ldots \mu_{k+1}} \in \mathcal{A}_{\star}$, we get for some constants $C_{0}(f), \ldots, C_{d}(f)$ depending on $f$ and on the choice of factorization at each step:

$$
\begin{aligned}
\left\|L_{\star}\left(\partial_{\mu} f\right) e^{-t \mathfrak{D}^{2}}\right\|_{1} \leq & \sum_{k=0}^{d-1} C_{k}(f) t^{k / 2} \max \left(t^{-\frac{d}{2}}, 1\right) \\
& +C_{d}(f) t^{\frac{d}{2}} \sum_{\mu_{1}, \ldots, \mu_{d}=1}^{d}\left\|L_{\star}\left(f_{2, \mu_{1} \ldots \mu_{d}}\right) e^{-t \mathfrak{D}_{\bullet}^{2} / 4^{d}}\right\|_{1} .
\end{aligned}
$$

Using Lemma 9 we get

$$
\begin{aligned}
\left\|L_{\star}\left(f_{2, \mu_{1} \ldots, \mu_{d}}\right) e^{-t \mathscr{D}_{\bullet}^{2} / 4^{d}}\right\|_{1} & \leq\left\|L_{\star}\left(f_{2, \mu_{1} \ldots, \mu_{d}}\right)\right\|\left\|e^{-t \mathscr{D}_{\bullet}^{2} / 4^{d}}\right\|_{1} \\
& \leq C^{\prime \prime}\left\|f_{2, \mu_{1}, \ldots, \mu_{d}}\right\|_{2} \max \left(t^{-d}, 1\right),
\end{aligned}
$$

which completes the proof. 
Corollary 13. For any $f \in \mathcal{A}_{\star}$, the operator $\left[\left(\mathscr{D}_{\bullet}^{2}+1\right)^{-\frac{d}{2}}, L_{\star}(f)\right]$ is of trace class.

Proof. By factorization $f=f_{1} \star f_{2}, f_{1}, f_{2} \in \mathcal{A}_{\star}$ and Leibniz rule,

$$
\begin{aligned}
& {\left[\left(\mathcal{D}_{\bullet}^{2}+1\right)^{-\frac{d}{2}}, L_{\star}(f)\right]} \\
& \quad=L_{\star}\left(f_{1}\right)\left[\left(\mathcal{D}_{\bullet}^{2}+1\right)^{-\frac{d}{2}}, L_{\star}\left(f_{2}\right)\right]-\left(L_{\star}\left(\bar{f}_{2}\right)\left[\left(\mathcal{D}_{\bullet}^{2}+1\right)^{-\frac{d}{2}}, L_{\star}\left(\bar{f}_{1}\right)\right]\right)^{*},
\end{aligned}
$$

it suffices to show that $L_{\star}\left(f_{1}\right)\left[\left(\mathscr{D}_{\bullet}^{2}+1\right)^{-\frac{d}{2}}, L_{\star}\left(f_{2}\right)\right]$ is of trace class for arbitrary $f_{1}, f_{2} \in \mathcal{A}_{\star}$. By spectral theory,

$$
L_{\star}\left(f_{1}\right)\left[\left(\mathscr{D}_{\bullet}^{2}+1\right)^{-\frac{d}{2}}, L_{\star}\left(f_{2}\right)\right]=\frac{1}{\Gamma\left(\frac{d}{2}\right)} \int_{0}^{\infty} d t t^{\frac{d}{2}-1} L_{\star}\left(f_{1}\right)\left[e^{-t\left(\mathscr{D}_{\bullet}^{2}+1\right)}, L_{\star}\left(f_{2}\right)\right] .
$$

Combining Lemma 11 with Lemma 12 we obtain for a finite constant depending only on $f$ :

$$
\left\|L_{\star}\left(f_{1}\right)\left[\left(\mathscr{D}_{\bullet}^{2}+1\right)^{-\frac{d}{2}}, L_{\star}\left(f_{2}\right)\right]\right\|_{1} \leq C(f) \int_{0}^{\infty} d t e^{-t} t^{\frac{d}{2}-1} \cdot t^{\frac{1}{2}} \cdot \max \left(t^{-\frac{d}{2}}, t^{\frac{d}{2}}\right) .
$$

As the integral converges, we are done.

We have arrived at the main result of this subsection, that the spectral triple $\left(\mathcal{A}_{\star}, \mathscr{H}, \mathscr{D}_{\bullet}\right)$ has metric dimension $d$ and not $2 d$ (remember that $d$ is even).

Theorem 14. For $f \in \mathcal{A}_{\star}, \bullet=1,2$, the operator $L_{\star}(f)\left(1+\mathscr{D}_{\bullet}^{2}\right)^{-\frac{d}{2}}$ belongs to $\mathscr{L}^{1, \infty}(\mathscr{H})$ and for any Dixmier trace $\operatorname{Tr}_{\omega}$, we have

$$
\operatorname{Tr}_{\omega}\left(L_{\star}(f)\left(1+\mathscr{D}_{\bullet}^{2}\right)^{-\frac{d}{2}}\right)=\frac{1}{\pi^{\frac{d}{2}}\left(\frac{d}{2}\right) !} \int d x \sqrt{\operatorname{det} g} f(x) .
$$

Proof. We use the factorization property (7) to write $f=f_{1} \star f_{2}$ with $f_{1}, f_{2} \in \mathcal{A}_{\star}$, which gives

$$
\begin{aligned}
& L_{\star}(f)\left(1+\mathscr{D}_{\bullet}^{2}\right)^{-\frac{d}{2}} \\
& \quad=L_{\star}\left(f_{1}\right)\left(1+\mathscr{D}_{\bullet}^{2}\right)^{-\frac{d}{2}} L_{\star}\left(f_{2}\right)+L_{\star}\left(f_{1}\right)\left[L_{\star}\left(f_{2}\right),\left(1+\mathcal{D}_{\bullet}^{2}\right)^{-\frac{d}{2}}\right] .
\end{aligned}
$$

First, by Corollary $13, L_{\star}\left(f_{1}\right)\left[L_{\star}\left(f_{2}\right),\left(1+\mathscr{D}_{\bullet}^{2}\right)^{-\frac{d}{2}}\right]$ is trace class, in particular it belongs to $\mathscr{L}^{1, \infty}(\mathscr{H})$. Then by Lemma 7 we get that $\operatorname{Tr}\left(L_{\star}(f) e^{-t \mathscr{D}_{\bullet}^{2}} L_{\star}(f)\right) \leq$ $C t^{-\frac{d}{2}}$, for all $f \in \mathcal{A}_{\star}$. This implies by [4], Theorem 4.4, that $L_{\star}(f) \in \mathcal{B}_{\zeta}\left(\left|D_{\bullet}\right|^{-\frac{1}{2}}\right)$ (see [4], Definition 4.2 and Proposition 4.3, for the definition of $\mathscr{B}_{\zeta}\left(\left|D_{\bullet}\right|^{-\frac{1}{2}}\right)$ ). Then [4], Proposition 4.8, ensures that $L_{\star}\left(f_{1}\right)\left(1+\mathscr{D}_{\bullet}^{2}\right)^{-\frac{d}{2}} L_{\star}\left(f_{2}\right)$ belongs to $\mathscr{L}^{1, \infty}(\mathscr{H})$. Therefore, $L_{\star}(f)\left(1+\mathscr{D}_{\bullet}^{2}\right)^{-\frac{d}{2}}$ is Dixmier-trace-class too, and any of its Dixmier trace coincides with those of $L_{\star}\left(f_{1}\right)\left(1+\mathscr{D}_{\bullet}^{2}\right)^{-\frac{d}{2}} L_{\star}\left(f_{2}\right)$. 
Using a polarization identity, it suffices to compute

$$
\operatorname{Tr}_{\omega}\left(L_{\star}(\bar{f})\left(1+D_{\bullet}^{2}\right)^{-\frac{d}{2}} L_{\star}(f)\right),
$$

for $f \in \mathcal{A}_{\star}$. Note that

$$
\begin{aligned}
\lim _{s \rightarrow 1^{+}}(s & -1) \operatorname{Tr}\left(L_{\star}(\bar{f})\left(1+\mathcal{D}_{\bullet}^{2}\right)^{-\frac{d s}{2}} L_{\star}(f)\right) \\
& =\lim _{s \rightarrow 1^{+}} \frac{s-1}{\Gamma\left(\frac{d s}{2}\right)} \int_{0}^{\infty} d t e^{-t} t^{\frac{d s}{2-1}} \operatorname{Tr}\left(L_{\star}(\bar{f}) e^{-t \mathscr{D}_{\bullet}^{2}} L_{\star}(f)\right) \\
& =\lim _{s \rightarrow 1^{+}} \frac{s-1}{\Gamma\left(\frac{d s}{2}\right)} \int_{0}^{\infty} d t e^{-t} t^{\frac{d(s-1)}{2-1}} \frac{(\tilde{\Omega} t)^{\frac{d}{2}}}{\pi^{\frac{d}{2}} \tanh ^{\frac{d}{2}}(\tilde{\Omega} t)} \\
& \cdot \int d z \sqrt{\operatorname{det} g} \bar{f} \star f(z) e^{-\tilde{\Omega} \tanh (\tilde{\Omega} t)\langle z, g z\rangle} \\
& =\frac{1}{\pi^{\frac{d}{2}}} \lim _{s \rightarrow 1^{+}} \frac{(s-1) \Gamma\left(\frac{d(s-1)}{2}\right)}{\Gamma\left(\frac{d s}{2}\right)} \int d z \sqrt{\operatorname{det} g} \bar{f} \star f(z) .
\end{aligned}
$$

Now [4], Proposition 5.13, which relies on Corollary 13, gives for any Dixmier trace

$$
\operatorname{Tr}_{\omega}\left(L_{\star}(\bar{f})\left(1+D_{\bullet}^{2}\right)^{-\frac{d}{2}} L_{\star}(f)\right)=\frac{1}{\pi^{\frac{d}{2}\left(\frac{d}{2}\right) !}} \int d x \sqrt{\operatorname{det} g} \bar{f} \star f(x) .
$$

This is all what we needed to prove.

2.6. Regularity and dimension spectrum. Our next task is to check the regularity condition. For that we relate the unbounded operator $\delta_{\bullet}$ defined by $\delta_{\bullet}(T):=$ $\left[\left\langle D_{\bullet}\right\rangle, T\right]$, with $\left\langle\mathcal{D}_{\bullet}\right\rangle:=\left(\mathcal{D}_{\bullet}^{2}+1\right)^{\frac{1}{2}}$, to the unbounded operator $R_{\bullet}$ defined by

$$
R_{\bullet}(T):=\left[\mathscr{D}_{\bullet}^{2}, T\right]\left\langle\mathcal{D}_{\bullet}\right\rangle^{-1}
$$

From the spectral representation of a positive operator $A$ :

$$
A=\frac{1}{\pi} \int_{0}^{\infty} d \lambda \lambda^{-\frac{1}{2}} \frac{A^{2}}{A^{2}+\lambda}
$$

we get the identity

$$
\delta_{\bullet}(T)=\frac{1}{\pi} \int_{0}^{\infty} d \lambda \lambda^{\frac{1}{2}} \frac{1}{\left\langle\mathcal{D}_{\bullet}\right\rangle^{2}+\lambda}\left[\left\langle\mathscr{D}_{\bullet}\right\rangle^{2}, T\right] \frac{1}{\left\langle\mathcal{D}_{\bullet}\right\rangle^{2}+\lambda} .
$$

Commuting $\left[\left\langle\mathcal{D}_{\bullet}\right\rangle^{2}, b\right]$ with $\left(\left\langle\mathcal{D}_{\bullet}\right\rangle^{2}+\lambda\right)^{-1}$ to the left, we get after some re-arrangements and using $\int d \lambda \lambda^{\frac{1}{2}} t\left(t^{2}+\lambda\right)^{-2}=\pi / 2$ :

$$
\delta_{\bullet}(T)=\frac{1}{2} R_{\bullet}(T)-\frac{1}{\pi} \int_{0}^{\infty} d \lambda \lambda^{\frac{1}{2}} \frac{1}{\left\langle\mathcal{D}_{\bullet}\right\rangle^{2}+\lambda} R_{\bullet}^{2}(T) \frac{\left\langle\mathscr{D}_{\bullet}\right\rangle^{2}}{\left(\left\langle\mathcal{D}_{\bullet}\right\rangle^{2}+\lambda\right)^{2}} .
$$


This suggests to introduce the map $\mathcal{T}_{\bullet}: \mathcal{B}(\mathscr{H}) \rightarrow \mathscr{B}(\mathscr{H})$ given by

$$
T \mapsto \mathcal{T}_{\bullet}(T):=\frac{2}{\pi} \int_{0}^{\infty} d \lambda \lambda^{\frac{1}{2}} \frac{1}{\left\langle D_{\bullet}\right\rangle^{2}+\lambda} T \frac{\left\langle\mathscr{D}_{\bullet}\right\rangle^{2}}{\left(\left\langle\mathscr{D}_{\bullet}\right\rangle^{2}+\lambda\right)^{2}} .
$$

Note that this operator is contractive. Indeed

$$
\left\|\mathcal{T}_{\bullet}\right\| \leq \frac{2}{\pi} \int_{0}^{\infty} \frac{\lambda^{\frac{1}{2}}}{(1+\lambda)^{2}} d \lambda=1 .
$$

Thus $2 \delta_{\bullet}=R_{\bullet}-\mathcal{T}_{\bullet} \circ R_{\bullet}^{2}$ and since $\mathcal{T}_{\bullet}$ commutes with $R_{\bullet}$ (because $R_{\bullet}$ commutes with the operators of left and right multiplications by functions of $\left.\left\langle\mathscr{D}_{\bullet}\right\rangle\right)$, we get

$$
2^{n} \delta_{\bullet}^{n}=\sum_{k=0}^{n}\left(\begin{array}{l}
n \\
k
\end{array}\right)(-1)^{k} \mathcal{T}_{\bullet}^{k} \circ R_{\bullet}^{n+k} .
$$

As a corollary ${ }^{1}$ we obtain $\bigcap_{n=1}^{\infty} \operatorname{dom}\left(\delta_{\bullet}^{n}\right) \supset \bigcap_{n=1}^{\infty} \operatorname{dom}\left(R_{\bullet}^{n}\right)$. With these preparations we prove:

Proposition 15. For any $f \in \mathscr{B}_{\star}$ and $\bullet=1,2$, both $L_{\star}(f)$ and $\left[\mathscr{D}_{\bullet}, L_{\star}(f)\right]$ belong to $\bigcap_{n=1}^{\infty} \operatorname{dom} \delta_{\bullet}^{n}$, where $\delta_{\bullet}(T):=\left[\left\langle\mathscr{D}_{\bullet}\right\rangle, T\right]$ and $\left\langle\mathscr{D}_{\bullet}\right\rangle:=\left(\mathscr{D}_{\bullet}^{2}+1\right)^{\frac{1}{2}}$.

Proof. Let $T=\left[\mathscr{D}_{1}, L_{\star}(f)\right]=\Gamma^{\mu} L_{\star}\left(i \partial_{\mu} f\right)$. It follows from $\left(\operatorname{ad}\left(\mathscr{D}_{1}^{2}\right)\right)^{n}\left(\Gamma^{\mu}\right)=$ $(\operatorname{ad}(\widetilde{\Omega} \Sigma))^{n}\left(\Gamma^{\mu}\right)$ that $R_{1}^{n}\left(\Gamma^{\mu}\right)$ is bounded for any $n$, and similarly for $R_{2}^{n}\left(\Gamma^{d+\mu}\right)$. Since $\mathcal{T}_{\bullet}$ is contractive, it follows from (17) that $\delta_{1}^{n}\left(\Gamma^{\mu}\right)$ and $\delta_{2}^{n}\left(\Gamma^{d+\mu}\right)$ are bounded. Hence, it suffices to show $L_{\star}(f) \in \bigcap_{n=1}^{\infty} \operatorname{dom}\left(R_{\bullet}^{n}\right)$. Since $\mathscr{D}_{\bullet}^{2}=H-(-1)^{\bullet} \widetilde{\Omega} \Sigma$ and because $\Sigma$ commutes with $H$ and with $L_{\star}(f)$, we get

$$
R_{\bullet}^{n}\left(L_{\star}(f)\right)=\left\langle D_{\bullet}\right\rangle^{-n}(\operatorname{ad}(H))^{n}\left(L_{\star}(f)\right)
$$

Now Lemma 4 shows that $(\operatorname{ad}(H))^{n}\left(L_{\star}(f)\right)$ can be written as a sum of terms of the form $\hat{\nabla}^{k} L_{\star}\left(f_{k}\right)$ with $f_{k} \in \mathscr{B}_{\star}$ and $k \leq n$, where $\hat{\nabla}$ is $\nabla$ or $\tilde{\nabla}$. Then one concludes using Corollary 5.

In the next and in analogy with the regularity condition, we will prove that one can determine the dimension spectrum with the operator $R_{\bullet}$ instead of $\delta$.

Proposition 16. Suppose that $b$ belongs to the polynomial algebra generated by $\delta_{\bullet}^{n}\left(\mathcal{A}_{\star}\right)$ and $\delta_{\bullet}^{n}\left(\left[\mathscr{D}_{\bullet} \mathcal{A}_{\star}\right]\right)$. Let $\zeta_{b}(z):=\operatorname{Tr}\left(b\left\langle\mathscr{D}_{\bullet}\right\rangle^{-z}\right)$, defined on the open half plane $\operatorname{Re}(z)>2 d$. Then, for all $M \in \mathbb{R}, \zeta_{b}$ is a finite sum of terms of the form $\operatorname{Tr}\left(R_{\bullet}^{n_{1}}\left(b_{1}\right) \ldots R_{\bullet}^{n_{k}}\left(b_{k}\right)\left\langle\mathscr{D}_{\bullet}\right\rangle^{-z-m}\right), n_{j}, k, m \in \mathbb{N}, b_{j} \in \mathcal{A}_{\star} \cup\left[\mathscr{D}_{\bullet}, \mathcal{A}_{\star}\right]$, plus $a$ function holomorphic on the half plane $\operatorname{Re}(z)>M$.

\footnotetext{
${ }^{1}$ In fact one has $\bigcap_{n=1}^{\infty} \operatorname{dom}\left(\delta_{\bullet}^{n}\right)=\bigcap_{n=1}^{\infty} \operatorname{dom}\left(R_{\bullet}^{n}\right)=\bigcap_{n=1}^{\infty} \operatorname{dom}\left(L_{\bullet}^{n}\right)$ with $L_{\bullet}(T):=$ $\left\langle D_{\bullet}\right\rangle^{-1}\left[\mathscr{D}_{\bullet}^{2}, T\right]$, see [3], eq. (2.8).
} 
Proof. According to (17), a typical element of the algebra generated by $\delta_{\bullet}^{n}\left(L_{\star}(f)\right)$ and $\delta_{\bullet}^{n}\left(\left[D_{\bullet}, L_{\star}(f)\right]\right)$, is a finite sum of elements of the form

$$
\prod_{j=1}^{k} \mathcal{T}_{\bullet}^{n_{j}} \circ R_{\bullet}^{m_{j}}\left(b_{j}\right), \quad b_{j} \in \mathcal{A}_{\star} \cup\left[\mathcal{D}_{\bullet}, \mathcal{A}_{\star}\right], n_{j}<m_{j} \in \mathbb{N} .
$$

For $\operatorname{Re}(z)>2 d,\left\langle D_{\bullet}\right\rangle^{-z}$ is a trace class operator. Since the algebra generated by $\delta_{\bullet}^{n}\left(L_{\star}(f)\right)$ and $\delta_{\bullet}^{n}\left(\left[\mathcal{D}_{\bullet}, L_{\star}(f)\right]\right)$ consists by Proposition 15 of bounded operators, $b\left\langle D_{\bullet}\right\rangle^{-z}$ is trace class for $\operatorname{Re}(z)>2 d$ and any $b$ in this polynomial algebra. For the same reason, the function

$$
\zeta_{R, \mathcal{T}}\left(b_{1}, n_{1}, m_{1} ; \ldots ; b_{k}, n_{k}, m_{k} ; z\right):=\operatorname{Tr}\left(\prod_{j=1}^{k} \mathcal{T}_{\bullet}^{n_{j}} \circ R_{\bullet}^{m_{j}}\left(b_{j}\right)\left\langle\mathcal{D}_{\bullet}\right\rangle^{-z}\right)
$$

is holomorphic on the open half plane $\operatorname{Re}(z)>2 d$. Starting from the definition, we have

$$
\begin{aligned}
& \prod_{j=1}^{k} \mathcal{T}_{\bullet}^{n_{j}} \circ R_{\bullet}^{m_{j}}\left(b_{j}\right) \\
& \quad=\int_{[0, \infty]^{|n|}} d \lambda^{|n|} \prod_{j=1}^{k}\left(\prod_{r_{j}=1}^{n_{j}} \frac{(2 / \pi) \lambda_{r_{j}}^{\frac{1}{2}}}{\left\langle\mathscr{D} \bullet \bullet_{\bullet}\right\rangle^{2}+\lambda_{r_{j}}}\right) R_{\bullet}^{m_{j}}\left(b_{j}\right)\left(\prod_{s_{j}=1}^{n_{j}} \frac{\left\langle\mathscr{D}_{\bullet}\right\rangle^{2}}{\left(\left\langle\mathcal{D}_{\bullet}\right\rangle^{2}+\lambda_{s_{j}}\right)^{2}}\right) .
\end{aligned}
$$

The next step consists in commuting for each $j$ the $R_{\bullet}^{m_{j}}\left(b_{j}\right)$ to the left of $\left(\left\langle\mathcal{D}_{\bullet}\right\rangle^{2}+\right.$ $\left.\lambda_{r_{j}}\right)^{-1}$ :

$$
\begin{aligned}
& {\left[\frac{1}{\left\langle\mathscr{D}_{\bullet}\right\rangle^{2}+\lambda_{j}}, R_{\bullet}^{m_{j}}\left(b_{j}\right)\right]=-\frac{1}{\left\langle\mathscr{D}_{\bullet}\right\rangle^{2}+\lambda_{j}} R_{\bullet}^{m_{j}+1}\left(b_{j}\right) \frac{\left\langle\mathscr{D}_{\bullet}\right\rangle}{\left\langle\mathcal{D}_{\bullet}\right\rangle^{2}+\lambda_{j}}} \\
& =\sum_{p_{j}=1}^{N_{j}}(-1)^{p_{j}} R_{\bullet}^{m_{j}+p_{j}}\left(b_{j}\right) \frac{\left\langle\mathcal{D}_{\bullet}\right\rangle^{p_{j}}}{\left(\left\langle\mathcal{D}_{\bullet}\right\rangle^{2}+\lambda_{j}\right)^{p_{j}+1}} \\
& +\frac{(-1)^{N_{j}+1}}{\left\langle\mathscr{D}_{\bullet}\right\rangle^{2}+\lambda_{j}} R_{\bullet}^{m_{j}+N_{j}+1}\left(b_{j}\right)\left(\frac{\left\langle D_{\bullet}\right\rangle}{\left\langle D_{\bullet}\right\rangle^{2}+\lambda_{j}}\right)^{N_{j}+1} \text {. }
\end{aligned}
$$

Any of the resulting $\lambda$-integrals is convergent, and $R_{\bullet}^{n}(b)$ is bounded for all $n \in \mathbb{N}$. Choosing the $N_{j}$ large enough, we generate as much negative powers of $\left\langle D_{\bullet}\right\rangle$ as necessary to make the product of the remainder with $\left\langle\mathscr{D}_{\bullet}\right\rangle^{-z}$ a trace-class operator for any given $z$ with $\operatorname{Re}(z)>M$ (if $M$ gets more and more negative we need larger and larger $N_{j}$ ). The other terms integrate to

$$
\int_{[0, \infty]} d \lambda_{j} \frac{(2 / \pi) \lambda_{j}^{\frac{1}{2}}\left\langle\mathcal{D}_{\bullet}\right\rangle^{p_{j}+2}}{\left(\left\langle\mathscr{D}_{\bullet}\right\rangle^{2}+\lambda_{r_{j}}\right)^{p_{j}+3}}=\frac{\Gamma\left(\frac{3}{2}+p_{j}\right)\left\langle\mathscr{D}_{\bullet}\right\rangle^{-1-p_{j}}}{\sqrt{\pi} \Gamma\left(3+p_{j}\right)},
$$


so that, up to the remainder term, which is easily seen to be holomorphic on the open half plane $\operatorname{Re}(z)>M, \prod_{j=1}^{k} \mathcal{T}_{\bullet}^{n_{j}} R_{\bullet}^{m_{j}}\left(b_{j}\right)$ is a finite linear combination of

$$
R_{\bullet}^{m_{1}+q_{1}}\left(b_{1}\right)\left\langle\mathscr{D}_{\bullet}\right\rangle^{-n_{1}-q_{1}} R_{\bullet}^{m_{2}+q_{2}}\left(b_{2}\right)\left\langle\mathscr{D}_{\bullet}\right\rangle^{-n_{2}-q_{2}} \ldots R_{\bullet}^{m_{k}+q_{k}}\left(b_{k}\right)\left\langle\mathscr{D}_{\bullet}\right\rangle^{-n_{k}-q_{k}} .
$$

The final step consists in commuting the $\left\langle\mathscr{D}_{\bullet}\right\rangle^{-n_{j}-q_{j}}$ to the right. If $n_{j}+q_{j}=2 l_{j}$ is even, we use the ( $\left.\lambda_{j}=0\right)$-case of (18). If $n_{j}+q_{j}=2 l_{j}-1$ is odd,

$$
\begin{aligned}
& {\left[\left\langle\mathscr{D}_{\bullet}\right\rangle^{1-2 l_{j}}, R_{\bullet}^{m_{j+1}^{\prime}}\left(b_{j+1}\right)\right] } \\
& \quad=\left\langle\mathscr{D}_{\bullet}\right\rangle^{-2 l_{j}} \delta_{\bullet}\left(R_{\bullet}^{m_{j+1}^{\prime}}\left(b_{j+1}\right)\right)+\left[\left\langle\mathcal{D}_{\bullet}\right\rangle^{-2 l_{j}}, R_{\bullet}^{m_{j+1}^{\prime}}\left(b_{j+1}\right)\right]\left\langle\mathcal{D}_{\bullet}\right\rangle .
\end{aligned}
$$

Using $\delta_{\bullet}=\frac{1}{2}\left(R_{\bullet}-\mathcal{T}_{\bullet} \circ R_{\bullet}^{2}\right)$, this case is reduced to the first one. Eventually, we conclude that, up to a remainder term, which again is easily seen to be holomorphic on the open half plane $\operatorname{Re}(z)>M, \prod_{j=1}^{k} \mathcal{T}_{\bullet}^{n_{j}} \circ R_{\bullet}^{m_{j}}\left(b_{j}\right)$ is a finite linear combination of

$$
R_{\bullet}^{m_{1}^{\prime}}\left(b_{1}\right) R_{\bullet}^{m_{2}^{\prime}}\left(b_{2}\right) \ldots R_{\bullet}^{m_{k}}\left(b_{k}\right)\left\langle D_{\bullet}\right\rangle^{-m} .
$$

This concludes the proof.

We can now state the main result of this section, namely:

Theorem 17. For $\bullet=1,2$, the spectral triple $\left(\mathcal{A}_{\star}, \mathcal{H}, \mathscr{D}_{\bullet}\right)$ has dimension spectrum $\mathrm{Sd}=d-\mathbb{N}$. Moreover, all poles of $\zeta_{b}(z)$ at $z \in \mathrm{Sd}$ are simple with local residues, i.e., for $b=\delta_{\bullet}^{n_{1}} L_{\star}\left(f_{1}\right) \ldots \delta_{\bullet}^{n_{k}} L_{\star}\left(f_{k}\right)$, any residue $\operatorname{res}_{z \in \mathrm{Sd}} \zeta_{b}(z)$ is a finite sum of terms of the form

$$
\int_{\mathbb{R}^{d}} d x x^{\alpha_{0}} \star\left(\partial^{\alpha_{1}} f_{1}\right) \star \cdots \star\left(\partial^{\alpha_{k}} f_{k}\right),
$$

where $\alpha_{i} \in \mathbb{N}^{d}$. An analogous result holds when the $L_{\star}\left(f_{k}\right)$ 's in $b$ are replaced by $\left[D_{.}, L_{\star}\left(f_{k}\right)\right]$ 's.

Proof. According to Proposition 16, it is equivalent to consider the functions

$$
\operatorname{Tr}\left(R_{\bullet}^{m_{1}}\left(b_{1}\right) \ldots R_{\bullet}^{m_{k}}\left(b_{k}\right)\left\langle\mathscr{D}_{\bullet}\right\rangle^{-z}\right), \quad b_{i} \in \mathcal{A}_{\star} \cup\left[\mathscr{D}_{\bullet}, \mathcal{A}_{\star}\right],
$$

instead of $\zeta_{b}(z)$. These functions are well defined for $\operatorname{Re}(z)>2 d$, because $R_{\bullet}^{m}(b)$ is bounded.

Since $[H, \Sigma]=0$, we get from the definition

$$
R_{\bullet}^{m_{j}}\left(b_{j}\right)=\sum_{k=0}^{m_{j}}\left(\begin{array}{c}
m_{j} \\
k
\end{array}\right)(\operatorname{ad}(H))^{k}\left((\operatorname{ad}(\tilde{\Omega} \Sigma))^{m_{j}-k}\left(b_{j}\right)\right)\left\langle D_{\bullet}\right\rangle^{-m_{j}} .
$$

Since $b_{j}$ is either $L_{\star}\left(f_{j}\right)$ or $i \Gamma^{\mu} L_{\star}\left(\partial_{\mu} f_{j}\right)$ for $\bullet=1$ and $i \Gamma^{\mu+d} L_{\star}\left(\partial_{\mu} f_{j}\right)$ for $\bullet=2$, we get

$$
(\operatorname{ad}(\widetilde{\Omega} \Sigma))^{m_{j}-k}\left(b_{j}\right)=\left\{\begin{array}{l}
\delta_{k, m_{j}} L_{\star}\left(f_{j}\right), \\
(\operatorname{ad}(\widetilde{\Omega} \Sigma))^{m_{j}-k}\left(\Gamma^{\mu}\right) L_{\star}\left(i \partial_{\mu} f_{j}\right), \\
(\operatorname{ad}(\widetilde{\Omega} \Sigma))^{m_{j}-k}\left(\Gamma^{\mu+d}\right) L_{\star}\left(i \partial_{\mu} f_{j}\right) .
\end{array}\right.
$$


We may therefore assume that $b_{j}=M_{j} L_{\star}\left(f_{j}\right)$ with $f_{j} \in \mathcal{A}$ and $M_{j} \in \operatorname{Mat}_{2^{d}}(\mathbb{C})$. Thus, the product $R_{\bullet}^{m_{1}}\left(b_{1}\right) \ldots R_{\bullet}^{m_{k}}\left(b_{k}\right)$ can be expressed as a finite sum of terms of the form

$$
(\operatorname{ad}(H))^{n_{1}}\left(L_{\star}\left(f_{1}^{\prime}\right)\right) M_{1}\left\langle\mathcal{D}_{\bullet}\right\rangle^{-m_{1}} \ldots(\operatorname{ad}(H))^{n_{k}}\left(L_{\star}\left(f_{k}^{\prime}\right)\right) M_{k}\left\langle\mathcal{D}_{\bullet}\right\rangle^{-m_{k}}, \quad n_{j} \leq m_{j} .
$$

Using the table given in Lemma 4 we can express $R_{\bullet}^{m_{1}}\left(b_{1}\right) \ldots R_{\bullet}^{m_{k}}\left(b_{k}\right)\left\langle\mathcal{D}_{\bullet}\right\rangle^{-z}$ as a finite sum of terms

$$
\begin{aligned}
L_{\star}\left(\partial^{\alpha_{1}} f_{1}\right) M_{1} P_{\alpha_{1}}(\hat{\nabla})\left\langle\mathscr{D}_{\bullet}\right\rangle^{-m_{1}} \ldots L_{\star}\left(\partial^{\alpha_{k}} f_{k}\right) M_{k} P_{\alpha_{k}}(\hat{\nabla})\left\langle\mathcal{D}_{\bullet}\right\rangle^{-m_{k}}\left\langle\mathcal{D}_{\bullet}\right\rangle^{-z} \\
=\frac{1}{\Gamma\left(\frac{m_{1}}{2}\right) \ldots \Gamma\left(\frac{m_{k-1}}{2}\right) \Gamma\left(\frac{m_{k}+z}{2}\right)} \int_{0}^{\infty} d t_{1} \ldots d t_{k} t_{1}^{\frac{m_{1}}{2}-1} \ldots t_{k}^{\frac{m_{k}+z}{2}-1} \\
\quad \cdot L_{\star}\left(\partial^{\alpha_{1}} f_{1}\right) M_{1} P_{\alpha_{1}}(\hat{\nabla}) e^{-t_{1}\left(\mathscr{D}_{\bullet}^{2}+1\right)} \ldots L_{\star}\left(\partial^{\alpha_{k}} f_{k}\right) M_{k} P_{\alpha_{k}}(\hat{\nabla}) e^{-t_{k}\left(\mathscr{D}_{\bullet}^{2}+1\right)},
\end{aligned}
$$

where $P_{\alpha_{j}}(\hat{\nabla})$ is a polynomial in $\nabla, \tilde{\nabla}$ of degree $\left|\alpha_{j}\right| \leq m_{j}$ (the $\alpha_{j}$ are multi-indices). We assume here that $m_{j} \geq 1$ for all $j$, otherwise (for $m_{j}=0$ ) we omit the $t_{j}$-integral and $\left(\Gamma\left(\frac{m_{j}}{2}\right)\right)^{-1}$.

Using

$$
\left[e^{-t_{j}\left(\mathscr{D}_{\bullet}^{2}+1\right)}, T\right]=-t_{j} \int_{0}^{1} d s_{j} e^{-t_{j} s_{j}\left(\mathscr{D}_{\bullet}^{2}+1\right)}\left[\mathscr{D}_{\bullet}^{2}, T\right] e^{-t_{j}\left(1-s_{j}\right)\left(\mathscr{D}_{\bullet}^{2}+1\right)},
$$

we commute all heat operators $e^{-t_{j}\left(\mathscr{D}_{\bullet}^{2}+1\right)}$ to the right, producing in each step a factor of $t_{j}$. The commutators $[H, T]$ are expressed by Lemma 4 and produce in each step at most one derivative $\nabla$. In the terms with all heat operators already on the right we then commute the derivatives $\hat{\nabla}$ to the right of all functions $f_{j}$ but left of all heat operators. The result is a finite sum of terms (with redefined $f_{j}, M_{j}$ )

$$
\begin{aligned}
& X= \frac{\int_{[0,1]^{N}} d s P(s)}{\Gamma\left(\frac{m_{1}}{2}\right) \ldots \Gamma\left(\frac{m_{k-1}}{2}\right) \Gamma\left(\frac{m_{k}+z}{2}\right)} \\
& \int_{0}^{\infty} d t_{1} \ldots d t_{k} t_{1}^{\frac{m_{1}}{2}+\beta_{1}-1} \ldots t_{k-1}^{\frac{m_{k-1}}{2}+\beta_{k-1}-1} t_{k}^{\frac{m_{k}+z}{2}+\beta_{k}-1} e^{-\left(t_{1}+\cdots+t_{k}\right)} \\
& \cdot\left(M_{1} \ldots M_{k} e^{(-)^{\bullet} \tilde{\Omega}\left(t_{1}+\cdots+t_{k}\right) \Sigma}\right) L_{\star}\left(\partial^{\gamma_{1}} f_{1} \star \ldots\right. \\
&\left.\ldots \star \partial^{\gamma_{k}} f_{k}\right) P_{\gamma_{1}, \ldots, \gamma_{k}}(\widehat{\nabla}) e^{-\left(t_{1}+\cdots+t_{k}\right) H},
\end{aligned}
$$

with $|m+\beta| \geq|\gamma|$ and $|\beta| \leq K$ ( $K$ can be chosen as big as one wishes by pushing the expansion far enough), plus a finite sum of remainders (with redefined $f_{j}, M_{j}$ )

$$
\begin{aligned}
Y= & \frac{1}{\Gamma\left(\frac{m_{1}}{2}\right) \ldots \Gamma\left(\frac{m_{k-1}}{2}\right) \Gamma\left(\frac{m_{k}+z}{2}\right)} \\
& \int_{0}^{\infty} d t_{1} \ldots d t_{k} t_{1}^{\frac{m_{1}}{2}+\beta_{1}^{\prime}-1} \ldots t_{k-1}^{\frac{m_{k-1}}{2}+\beta_{k-1}^{\prime}-1} t_{k}^{\frac{m_{k}+z}{2}+\beta_{k}^{\prime}-1} \\
& \cdot \int_{[0,1]^{N^{\prime}}} d s P^{\prime}(s) \prod_{j=1}^{k} L_{\star}\left(\partial^{\gamma_{j}^{\prime}} f_{j}\right) M_{j} P_{\gamma_{j}^{\prime}}(\hat{\nabla}) e^{-\tau_{j}\left(D_{\bullet}^{2}+1\right)},
\end{aligned}
$$


with $\left|\gamma^{\prime}\right| \leq\left|m+\beta^{\prime}\right|$ and $\left|\beta^{\prime}\right|>K$, where $\tau_{j}$ are positive functions of $\{s\}, t_{1}, \ldots, t_{k}$ with $\sum_{j=1}^{k} \tau_{j}=t_{1}+\cdots+t_{k}$.

In (19) we can use Lemma 4 to express $P_{\gamma}(\hat{\nabla})$ as a finite sum of $P_{\gamma^{\prime}}(x) P_{\gamma^{\prime \prime}}(\nabla)$. The polynomial $P_{\gamma}^{\prime}(x)$ can (under the trace) be moved into $L_{\star}(f)$. We change the variables $t_{1}=t \prod_{l=2}^{k}\left(1-u_{l}\right)$ and $t_{j}=t u_{j} \prod_{l=j+1}^{k}\left(1-u_{l}\right)$ for $j \geq 2$ with Jacobian $t^{k-1} \prod_{l=3}^{k}\left(1-u_{l}\right)^{l-2}$ and obtain

$$
\begin{aligned}
\operatorname{Tr}(X)= & \frac{\int_{[0,1]^{N}} d s P(s)}{\Gamma\left(\frac{|m|+z}{2}+|\beta|\right)}\left(\prod_{j=1}^{k-1} \frac{\Gamma\left(\frac{m_{j}}{2}+\beta_{j}\right)}{\Gamma\left(\frac{m_{j}}{2}\right)}\right) \frac{\Gamma\left(\frac{m_{k}+z}{2}+\beta_{k}\right)}{\Gamma\left(\frac{m_{k}+z}{2}\right)} \\
& \int_{0}^{\infty} d t e^{-t} t^{\frac{|m|+z}{2}+|\beta|-1} \operatorname{tr}_{\mathbb{C}^{2} d}\left(M_{1} \ldots M_{k} e^{(-1)^{\bullet} \widetilde{\Omega} t \Sigma}\right) \\
& \cdot \operatorname{Tr}\left(L_{\star}\left(x^{\gamma_{0}} \star \partial^{\gamma_{1}} f_{1} \star \cdots \star \partial^{\gamma_{k}} f_{k}\right) P_{\gamma_{1}, \ldots, \gamma_{k}}(\nabla) e^{-t H}\right) .
\end{aligned}
$$

Note that $\frac{\Gamma\left(\frac{m_{k}+z}{2}+\beta_{k}\right)}{\Gamma\left(\frac{m_{k}+z}{2}\right)}$ is a polynomial in $z$ of degree $\beta_{k}$.

The traces $\operatorname{Tr}\left(L_{\star}(f) \nabla_{\mu_{1}} \ldots \nabla_{\mu_{r}} e^{-t H}\right)$ are computed in Proposition 6. Accordingly, they have, up to a remainder which leads to a holomorphic function in $z$, an asymptotic expansion

$$
\operatorname{Tr}\left(L_{\star}(f) \nabla_{\mu_{1}} \ldots \nabla_{\mu_{|\gamma|}} e^{-t H}\right)=\sum_{a=0}^{N^{\prime \prime}} t^{-\frac{d}{2}-\left[\frac{|\gamma|}{2}\right]+a} \int_{\mathbb{R}^{d}} d x f(x) P_{|\gamma|}^{a}(x),
$$

where $P_{|\gamma|}^{a}(x)$ is a $\star$-polynomial of degree $\leq|\gamma|+2 a$. Inserted into (21), the $t$ integral of any such term yields (together with $\operatorname{tr}_{\mathbb{C}^{2 d}}\left(M_{1} \ldots M_{k} e^{(-1)^{\bullet} \tilde{\Omega} t \Sigma}\right)$ ) a linear combination of

$$
\frac{\Gamma\left(\frac{|m|+2|\beta|-2[|\gamma| / 2]+2 a+z-d}{2}\right)}{\Gamma\left(\frac{|m|+2 \beta+z}{2}\right)} \int_{\mathbb{R}^{d}} d x f(x) P_{|\gamma|}^{a}(x) .
$$

As a function of $z \in \mathbb{C}$, the latter is trivially holomorphic in $\mathbb{C} \backslash \mathbb{Z}$. As $|m|+|\beta| \geq|\gamma|$, this function is also holomorphic for $z \in \mathbb{N}$ with $z>d$. If $z=d-N$ for $N \in \mathbb{N}$, then there is a finite number of parameters $\beta_{j}, m_{j}, a, \gamma_{j}$ for which $\frac{|m|+2|\beta|-2[|\gamma| / 2]+2 a-N}{2}$ is a non-positive integer smaller than $\frac{|m|+2 \beta+d-N}{2}$. Precisely these parameters yield simple poles at $z=d-N$. Each residue has the claimed structure.

We estimate the remainders (20) in trace norm by

$$
\begin{gathered}
\frac{\left\|M_{1}\right\| \ldots\left\|M_{k}\right\|}{\left|\Gamma\left(\frac{m_{1}}{2}\right) \ldots \Gamma\left(\frac{m_{k}+z}{2}\right)\right|} \int_{0}^{\infty} d t_{1} \ldots d t_{k} t_{1}^{\frac{m_{1}}{2}+\beta_{1}^{\prime}-1} \ldots t_{k-1}^{\frac{m_{k-1}}{2}+\beta_{k-1}^{\prime}-1} t_{k}^{\frac{m_{k}+z}{2}+\beta_{k}^{\prime}-1} \\
\cdot \int_{[0,1]^{N^{\prime}}} d s\left|P^{\prime}(s)\right|\left\|\prod_{j=1}^{k} L_{\star}\left(\partial^{\gamma_{j}^{\prime}} f_{j}\right) e^{(-1)^{\bullet} \tau_{j} \tilde{\Omega} \Sigma} P_{\gamma_{j}^{\prime}}(\nabla) e^{-\tau_{j}(H+1)}\right\|_{1}
\end{gathered}
$$


By spectral theory and Corollary 5 , we get for $p \in[1, \infty)$ :

$$
\left\|P_{\gamma_{j}^{\prime}}(\nabla) e^{-\tau_{j}(H+1)}\right\|_{p} \leq C\left(\varepsilon \tau_{j}\right)^{-\gamma_{j}^{\prime} / 2}\left\|e^{-\tau_{j}^{\prime}(H+1)(1-\varepsilon)}\right\|_{p}
$$

Since the $\tau_{j}$ are linear in $t_{j}$ with $\sum_{j=1}^{k} \tau_{j}=\sum_{j=1}^{k} t_{j}$, setting $t=\sum_{j=1}^{k} t_{j}$, the Hölder inequality gives

$$
\begin{aligned}
& \left\|\prod_{j=1}^{k} L_{\star}\left(\partial^{\gamma_{j}^{\prime}} f_{j}\right) e^{(-1)^{\bullet} \tau_{j} \tilde{\Omega} \Sigma} P_{\gamma_{j}^{\prime}}(\nabla) e^{-\tau_{j}^{\prime}(H+1)}\right\|_{1} \\
& \leq \prod_{j=1}^{k}\left\|L_{\star}\left(\partial^{\gamma_{j}^{\prime}} f_{j}\right)\right\|\left\|e^{(-1)^{\bullet} \tau_{j} \tilde{\Omega} \Sigma}\right\|_{t / \tau_{j}}\left\|P_{\gamma_{j}^{\prime}}(\nabla) e^{-\tau_{j}(H+1)}\right\|_{t / \tau_{j}^{\prime} j} \\
& \leq C e^{-t(1-\varepsilon)} \prod_{j=1}^{k}\left(\varepsilon t_{j}\right)^{-\gamma_{j}^{\prime} / 2}\left\|L_{\star}\left(\partial^{\gamma_{j}^{\prime}} f_{j}\right)\right\|(2 \cosh (\widetilde{\Omega} t))^{\frac{d \tau_{j}}{t}}\left(2 \sinh (\widetilde{\Omega} t(1-\varepsilon))^{-\frac{d \tau_{j}}{t}}\right. \\
& =C e^{-t(1-\varepsilon-\tilde{\Omega} \varepsilon d)}\left(\prod_{j=1}^{k}\left(\varepsilon t_{j}\right)^{-\gamma_{j}^{\prime} / 2}\left\|L_{\star}\left(\partial^{\gamma_{j}^{\prime}} f_{j}\right)\right\|\right)\left(\frac{\cosh (\widetilde{\Omega} t)}{\sinh (\widetilde{\Omega} t(1-\varepsilon)) e^{\tilde{\Omega} t \varepsilon}}\right)^{d},
\end{aligned}
$$

where results of Proposition 6 and Lemma 7 have been used. Hence for $\varepsilon^{\prime}:=$ $(1-\varepsilon-\widetilde{\Omega} \varepsilon d)>0$ the remainders (20) are bounded in trace norm by

$$
\begin{aligned}
& \frac{C^{\prime}}{\left|\Gamma\left(\frac{m_{1}}{2}\right) \ldots \Gamma\left(\frac{m_{k}+z}{2}\right)\right|} \\
& \cdot \int_{0}^{\infty} d t_{1} \ldots d t_{k} t_{1}^{\frac{m_{1}}{2}+\beta_{1}^{\prime}-1-\frac{\gamma_{1}^{\prime}}{2}} \ldots t_{k}^{\frac{m_{k}+z}{2}+\beta_{k}^{\prime}-1-\frac{\gamma_{k}^{\prime}}{2}}\left(t_{1}+\cdots+t_{k}\right)^{-d} e^{-\varepsilon^{\prime}\left(t_{1}+\cdots+t_{k}\right)} .
\end{aligned}
$$

Remember that $\left|\gamma^{\prime}\right| \leq\left|\beta^{\prime}\right|+|m|$ and $\left|\beta^{\prime}\right|>K$ and that $K$ can be chosen as big as one wishes (by pushing the expansion over and over). So given $M \leq 2 d$, by choosing $K>M / 2+d$, we see that the remainder terms are well defined as traceclass operators for $\operatorname{Re}(z)>M$. A similar analysis involving the $z$-derivative of the remainders can be done, showing that by pushing the expansion far enough, the remainders yield holomorphic contributions for $\operatorname{Re}(z)>M$, with $M \in \mathbb{R}$ arbitrary.

\section{The spectral action}

3.1. Generalities. For a unital spectral triple with real structure $(\mathcal{A}, \mathscr{H}, \mathcal{D}, J)$, according to the spectral action principle [7], [5], the bosonic action should depend only on the spectrum of the fluctuated Dirac operator

$$
\mathscr{D} \mapsto \mathcal{D}_{A}:=\mathscr{D}+A+\varepsilon^{\prime} J A J^{-1},
$$


where $A=\sum a_{i}\left[\mathcal{D}, b_{i}\right], a_{i}, b_{i} \in \mathcal{A}$ (finite sum), is a self-adjoint one-form and $\varepsilon^{\prime}= \pm 1$ depending on the KO-dimension of the triple. Ideally, such an action functional (of $\mathscr{D}$ and of $A$ ) should be defined as the number of eigenvalues of $\mathscr{D}_{A}^{2}$ smaller than a given scale $\Lambda>0$ :

$$
S_{\Lambda}\left(\mathscr{D}_{A}\right)=\sharp\left\{\lambda_{n} \in \operatorname{Spec}\left(\mathscr{D}_{A}^{2}\right) \mid \lambda_{n} \leq \Lambda^{2}\right\}
$$

or akin to the same and with $\chi$ the characteristic function of the interval $[0,1]$ :

$$
S_{\Lambda}\left(\mathscr{D}_{A}\right)=\operatorname{Tr}\left(\chi\left(\mathcal{D}_{A}^{2} / \Lambda^{2}\right)\right) \text {. }
$$

Then the diverging part of $S_{\Lambda}\left(\mathscr{D}_{A}\right)$ in the limit $\Lambda \rightarrow \infty$ should give access to an effective action describing low energy physics. The problem is that with the characteristic function, the expression (22) may not have a well-defined power series expansion in the limit $\Lambda \rightarrow \infty$. To overcome this difficulty one uses, instead of the characteristic function, a smooth one approximating it and being the inverse Laplace transform of a Schwartz function on $\mathbb{R}_{+}^{*}$. By Laplace transformation one then has

$$
S_{\Lambda}\left(\mathscr{D}_{A}\right)=\int_{0}^{\infty} d t \operatorname{Tr}\left(e^{-t \mathscr{D}_{A}^{2} / \Lambda^{2}}\right) \hat{\chi}(t),
$$

where $\hat{\chi}$ is the inverse Laplace transform of $\chi$. Assuming that the trace of the heat kernel has an asymptotic expansion

$$
\operatorname{Tr}\left(e^{-t \mathscr{D}_{A}^{2}}\right)=\sum_{k=-n}^{\infty} a_{k}\left(\mathscr{D}_{A}^{2}\right) t^{k}, \quad n \in \mathbb{N},
$$

we obtain

$$
S_{\Lambda}\left(\mathscr{D}_{A}\right)=\sum_{k=-n}^{\infty} a_{k}\left(\mathscr{D}_{A}^{2}\right) \Lambda^{-2 k} \int_{0}^{\infty} d t t^{k} \hat{\chi}(t) .
$$

One easily finds

$$
\int_{0}^{\infty} d t t^{k} \hat{\chi}(t)= \begin{cases}\frac{1}{\Gamma(-k)} \int_{0}^{\infty} d s s^{-k-1} \chi(s) & \text { for } k \notin \mathbb{N} \\ (-1)^{k} \chi^{(k)}(0) & \text { for } k \in \mathbb{N}\end{cases}
$$

If one only wants to keep the non-vanishing terms in the power- $\Lambda$ expansion as $\Lambda \rightarrow \infty$, it is therefore sufficient to identify the non-vanishing terms in the power- $t$ expansion of $\operatorname{Tr}\left(e^{-t \mathscr{D}_{A}^{2}}\right)$ as $t \rightarrow 0$.

If the spectral geometry $(\mathcal{A}, \mathscr{H}, \mathscr{D}, J)$ is non-unital, then the expression (22) becomes ill-defined. There are different ways to 'regularize' the spectral action in this case. For instance one may consider instead

$$
S_{\Lambda}\left(\mathscr{D}_{A}\right):=\operatorname{Tr}\left(\chi\left(\mathcal{D}_{A}^{2} / \Lambda^{2}\right)-\chi\left(\mathscr{D}^{2} / \Lambda^{2}\right)\right) .
$$


But the problem is then that one loses a lot of physical information since one cannot access in this way the Einstein-Hilbert action. Another possibility, used in [19], is to introduce a supplementary (adynamic) scalar field $\rho \in \mathcal{A}$ and to define

$$
S_{\Lambda}\left(D_{A}, \rho\right):=\operatorname{Tr}\left(\rho \chi\left(\mathscr{D}_{A}^{2} / \Lambda^{2}\right)\right) .
$$

The advantage of this scheme is that one keeps the physical interpretation by performing on the field equations the adiabatic limit $\rho \rightarrow 1$ and that one can choose the one-form $A$ coming from the unitization of $\mathcal{A}$ and not necessarily from $\mathcal{A}$ itself. However, in the case of the Moyal spectral triple with ordinary Dirac operator, treated in [19], the full computation with the real structure was not possible; only the spectral action for partially fluctuated Dirac operator $\mathscr{D} \mapsto \mathscr{D}+A$ was evaluated. To our knowledge and up to now, there is only one situation of noncommutative spectral triple (excluding the almost commutative spectral triples) where the spectral action with fully perturbed Dirac operator has been computed. This is in the work of Essouabri-Iochum-Levy-Sitarz [17] for noncommutative tori.

The last possibility spelled out in [6] is to replace the scale $\Lambda$ by a dilaton field. That is, one performs the replacement

$$
\Lambda \mapsto e^{-\phi}, \quad \phi^{*}=\phi \in \mathcal{A},
$$

and one leaves (22) as it was:

$$
S_{\phi}\left(\mathscr{D}_{A}\right):=\operatorname{Tr}\left(\chi\left(e^{\phi} \mathscr{D}_{A}^{2} e^{\phi}\right)\right) .
$$

Although this expression is analytically well defined and conceptually perfect, the explicit computation of such a functional seems to be fairly inaccessible, except for the commutative (manifold) case.

In our setting of a spectral triple for Moyal plane with harmonic propagation, the question of the definition and the computation of the spectral action is way more easy. This is because although the spectral triple $\left(\mathscr{A}_{\star}, \mathcal{H}, \mathscr{D}_{\bullet}\right), \bullet=1,2$, is non-unital, the heat operator $e^{-t D_{\bullet}^{2}}$ is trace-class for all $t>0$ (see Lemma 9). This means that the definition (22) of the spectral action for unital spectral triple is still adapted to our situation.

In the next subsections we will perform a complete computation of the spectral action for a $U\left(\mathscr{B}_{\star}\right)$-Higgs model for $d=4$. Before this, we will derive a generic heat kernel type expansion when one tensorizes $\left(\mathcal{A}_{\star}, \mathscr{H}, \mathscr{D}_{\bullet}\right)$ with a finite spectral triple.

3.2. Heat kernel expansion in dimension four. We derive here a short-time heatkernel expansion for the semi-group generated by the square of a fluctuated harmonic Dirac operator, for the algebra of Schwartz functions with Moyal product. We start with preliminary results on Schatten norm estimates, using the estimate of Lemma 12 together with complex interpolation methods. Here we specify to the case $d=4$. 
Proposition 18. Let $f \in \mathcal{A}_{\star}$. Then for all $1 \leq p \leq \infty$ and $t \in(0,1]$ we have

$$
\left\|L_{\star}(f) e^{-t \mathscr{D}_{\bullet}^{2}}\right\|_{p} \leq C(\Theta)^{1-1 / p}\|f\|_{2}^{1-1 / p} C(f)^{1 / p} p^{-2 / p} t^{-2 / p},
$$

where $C(f)$ is the constant appearing in Lemma 12 and $C(\Theta)$ is the one appearing in (8).

Proof. For $f \in \mathcal{A}_{\star}, t \in(0,1]$ and $1 \leq p<\infty$, consider on the strip $S:=\{z \in \mathbb{C} \mid$ $\operatorname{Re}(z) \in[0,1]\}$ the operator-valued function

$$
F_{p}: z \mapsto L_{\star}(f) e^{-t p z \mathscr{D}_{\bullet}^{2}}
$$

The function $F_{p}$ is continuous on $S$, holomorphic on its interior and by Lemma 12 it satisfies for $y \in \mathbb{R}$ :

$$
\left\|F_{p}(i y)\right\| \leq C(\Theta)\|f\|_{2}, \quad\left\|F_{p}(1+i y)\right\|_{1} \leq C(f)(p t)^{-2} .
$$

Then by standard complex interpolation methods (see for example [35]) we have $F_{p}(z) \in \mathscr{L}^{1 / \operatorname{Re}(z)}(\mathscr{H})$ for all $z \in S$ with

$$
\begin{aligned}
\left\|F_{p}(z)\right\|_{1 / \operatorname{Re}(z)} & \leq\left\|F_{p}(0)\right\|_{\infty}^{1-\operatorname{Re}(z)}\left\|F_{p}(1)\right\|_{1}^{\operatorname{Re}(z)} \\
& \leq C(\Theta)^{1-\operatorname{Re}(z)}\|f\|_{2}^{1-\operatorname{Re}(z)} C(f)^{\operatorname{Re}(z)}(p t)^{-2 \operatorname{Re}(z)} .
\end{aligned}
$$

Applying this for $z=1 / p$, we get

$$
\left\|L_{\star}(f) e^{-t \mathscr{D}_{\bullet}^{2}}\right\|_{p} \leq C(\Theta)^{1-1 / p}\|f\|_{2}^{1-1 / p} C(f)^{1 / p} p^{-2 / p} t^{-2 / p},
$$

as needed.

Remark 19. For $f \in \mathcal{A}_{\star}$, making a recursive choice of factorization as follows:

$$
\begin{aligned}
& f=f_{1} \star f_{2},
\end{aligned}
$$

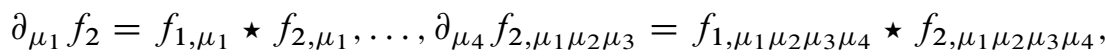

the constant $C(f)$ appearing in Lemma 12 (for $d=4$ ) and Proposition 18 is a finite 
multiple (depending only on $\widetilde{\Omega}$ and $\Theta$ ) of

$$
\begin{aligned}
& \left\|\bar{f}_{1} \star f_{1}\right\|_{1}^{\frac{1}{2}}\left\|\bar{f}_{2} \star f_{2}\right\|_{1}^{\frac{1}{2}} \\
& +\left\|f_{1}\right\|_{2} \sum_{\mu_{1}=1}^{4}\left(\left\|\bar{f}_{1, \mu_{1}} \star f_{1, \mu_{1}}\right\|_{1}^{\frac{1}{2}}\left\|\bar{f}_{2, \mu_{1}} \star f_{2, \mu_{1}}\right\|_{1}^{\frac{1}{2}}\right. \\
& +\left\|f_{1, \mu_{1}}\right\|_{2} \sum_{\mu_{2}=1}^{4}\left(\left\|\bar{f}_{1, \mu_{1} \mu_{2}} \star f_{1, \mu_{1} \mu_{2}}\right\|_{1}^{\frac{1}{2}}\left\|\bar{f}_{2, \mu_{1} \mu_{2}} \star f_{2, \mu_{1} \mu_{2}}\right\|_{1}^{\frac{1}{2}}\right. \\
& +\left\|f_{1, \mu_{1} \mu_{2}}\right\|_{2} \sum_{\mu_{3}=1}^{4}\left(\left\|\bar{f}_{1, \mu_{1} \mu_{2} \mu_{3}} \star f_{1, \mu_{1} \mu_{2} \mu_{3}}\right\|_{1}^{\frac{1}{2}}\left\|\bar{f}_{2, \mu_{1} \mu_{2} \mu_{3}} \star f_{2, \mu_{1} \mu_{2} \mu_{3}}\right\|_{1}^{\frac{1}{2}}\right. \\
& +\left\|f_{1, \mu_{1} \mu_{2} \mu_{3}}\right\|_{2} \sum_{\mu_{4}=1}^{4}\left(\left\|\bar{f}_{1, \mu_{1} \mu_{2} \mu_{3} \mu_{4}} \star f_{1, \mu_{1} \mu_{2} \mu_{3} \mu_{4}}\right\|_{1}^{\frac{1}{2}}\right.
\end{aligned}
$$

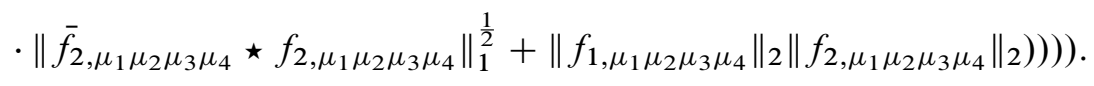

Lemma 20. Let $f \in \mathcal{A}_{\star}, 1 \leq p \leq \infty, t \in(0,1]$ and $k \in \mathbb{N}$. Then there exists $a$ finite constant $C_{p, k}(f)$ such that

$$
\left\|L_{\star}(f) \mathscr{D}_{\bullet}^{k} e^{-t \mathscr{D}_{\bullet}^{2}}\right\|_{p} \leq C_{p, k}(f) t^{-2 / p-k / 2} .
$$

Proof. By spectral theory, we have $\left\|\mathscr{D}_{\bullet}^{k} e^{-t \mathscr{D}_{\bullet}^{2}}\right\|=(k / 2 e t)^{k / 2}$, so the proof is a consequence of Proposition 18.

The next Lemma will explain why there is a major difference in the spectral action when perturbing $\mathscr{D}$ by $A+J A J^{-1}$ or simply by $A$.

Lemma 21. For $f, g \in \mathcal{A}_{\star}$, the operator $L_{\star}(f) R_{\star}(g)$ is of trace class on $\mathcal{H}$.

Proof. By factorization, we can find $f_{1}, f_{2}, g_{1}, g_{2} \in \mathcal{A}_{\star}$ such that

$$
f=f_{1} \star f_{2}, \quad g=g_{1} \star g_{2} .
$$

Hence

$$
L_{\star}(f) R_{\star}(g)=L_{\star}\left(f_{1} \star f_{2}\right) R_{\star}\left(g_{1} \star g_{2}\right)=L_{\star}\left(f_{1}\right) L_{\star}\left(f_{2}\right) R_{\star}\left(g_{1}\right) R_{\star}\left(g_{2}\right) .
$$

But since the left and right regular representations commute (by associativity of the Moyal product), we get

$$
L_{\star}(f) R_{\star}(g)=L_{\star}\left(f_{1}\right) R_{\star}\left(g_{1}\right) L_{\star}\left(f_{2}\right) R_{\star}\left(g_{2}\right),
$$

so that it suffices to show that $L_{\star}(f) R_{\star}(g)$ is Hilbert-Schmidt for all $f, g \in \mathcal{A}_{\star}$. From the operator kernel formula (14) of $L_{\star}(f)$, and a similar one for $R_{\star}(g)$, one 
easily deduces the operator kernel for the product $L_{\star}(f) R_{\star}(g)$, and after a few lines of computations, we get for a suitable constant depending only on $\operatorname{det}(\Theta)$ :

$$
\left\|L_{\star}(f) R_{\star}(g)\right\|_{2}^{2}=\int d x d y\left|\left[L_{\star}(f) R_{\star}(g)\right](x, y)\right|^{2}=C\|f\|_{2}^{2}\|g\|_{2}^{2} .
$$

This completes the proof.

Corollary 22. Let $\nabla_{\mu}^{a}, \mu=1, \ldots, 4$, be the operators on $L^{2}\left(\mathbb{R}^{4}\right)$ given by

$$
\nabla_{\mu}^{a}:=i \partial_{\mu}+a_{\mu \nu} x^{\mu}, \quad a \in M_{4}(\mathbb{R}) .
$$

Then for $f, g \in \mathcal{A}_{\star}, t \in(0,1]$ and $P_{\alpha}(\hat{\nabla})$ a polynomial of order $\alpha$ in the operators $\nabla_{\mu}^{a}$, there exists a finite constant $C(f, g, \alpha)$ such that

$$
\left\|L_{\star}(f) R_{\star}(g) P_{\alpha}(\hat{\nabla}) e^{-t H}\right\|_{1} \leq C(f, g, \alpha) t^{-\alpha / 2} .
$$

Proof. From Lemma 21, it suffices to show that $\left\|P_{\alpha}(\hat{\nabla}) e^{-t H}\right\| \leq C t^{-\alpha / 2}$, which will follow by spectral theory if $P_{\alpha}(\hat{\nabla})(1+H)^{-\alpha / 2}$ is bounded. But this is a slight generalization of Corollary 5.

We can now deduce the germ of the asymptotic expansion formula we need.

Proposition 23. Let $\left(\mathcal{A}_{\mathrm{f}}, \mathscr{H}_{\mathrm{f}}, \mathscr{D}_{\mathrm{f}}, J_{\mathrm{f}}\right)$ be a finite spectral triple. Let $\mathscr{D}:=D_{\bullet} \otimes 1+$ $\Gamma \otimes D_{\mathrm{f}}, \cdot=1,2$, be the Dirac operator of the product spectral triple $\left(\mathcal{A} \otimes \mathcal{A}_{\mathrm{f}}, \mathscr{H} \otimes \mathscr{H}_{\mathrm{f}}, \mathscr{D}_{\bullet} \otimes 1+\Gamma \otimes \mathscr{D}_{\mathrm{f}}\right)$. Let also $\mathscr{D}_{A}:=\mathscr{D}+A+\boldsymbol{J} A \boldsymbol{J}^{-1}$ be the fluctuated Dirac operator. Here $\boldsymbol{J}:=J \otimes J_{\mathrm{f}}$ and $A$ is a self-adjoint one-form, that is $A=A^{*}:=\sum_{i} a_{i}\left[D, b_{i}\right]$, where the sum is finite and $a_{i}, b_{i} \in \mathcal{A} \otimes \mathcal{A}_{\mathrm{f}}$. In terms of the decomposition $\mathscr{D}_{A}^{2}=\mathscr{D}^{2}+F_{0}+F_{1}+\boldsymbol{J}\left(F_{0}+F_{1}\right) \boldsymbol{J}^{-1}+2 A \boldsymbol{J} A \boldsymbol{J}^{-1}$, where $F_{0}$ is a bounded operator and $F_{1}$ is linear in the operators $\nabla_{\mu}^{a}$ of Corollary 22, the following holds:

$$
\begin{aligned}
\operatorname{Tr}\left(e^{\left.-t \mathscr{D}_{A}^{2}\right)=}\right. & \operatorname{Tr}\left(\left\{1-2 t\left(F_{0}+F_{1}\right)+t^{2}\left(F_{0}^{2}+F_{1} F_{0}+F_{0} F_{1}+F_{1}^{2}\right)\right.\right. \\
& -\frac{t^{3}}{3}\left(F_{0}\left[\mathscr{D}^{2}, F_{1}\right]-\left[\mathscr{D}^{2}, F_{1}\right] F_{0}+F_{1}\left[\mathscr{D}^{2}, F_{1}\right]+F_{0} F_{1}^{2}+F_{1} F_{0} F_{1}\right. \\
& \left.+F_{1}^{2} F_{0}+F_{1}^{3}\right)+\frac{t^{4}}{12}\left(F_{1}\left[\mathscr{D}^{2},\left[\mathscr{D}^{2}, F_{1}\right]\right]+2 F_{1}^{2}\left[\mathscr{D}^{2}, F_{1}\right]\right. \\
& \left.\left.\left.+F_{1}\left[\mathscr{D}^{2}, F_{1}\right] F_{1}+F_{1}^{4}\right)\right\} e^{-t \mathscr{D}^{2}}\right)+\mathcal{O}(\sqrt{t}) .
\end{aligned}
$$

Proof. First, it is clear that $e^{-t \mathscr{D}_{A}^{2}}$ is of trace-class for all $t>0$. Indeed, since the eigenvalues of $\mathscr{D}$. behave like $n^{\frac{1}{2}}$ with multiplicity which behaves like $n^{3}$, its resolvent belongs to the Schatten ideal $\mathscr{L}^{8+\varepsilon}(\mathscr{H})$ for all $\varepsilon>0$. From the relation

$$
\frac{1}{\mathscr{D}_{A}+i}=\frac{1}{\mathscr{D}_{\bullet} \otimes 1+i}\left(1-\left(A+\boldsymbol{J} A \boldsymbol{J}^{-1}+\Gamma \otimes \mathfrak{D}_{\mathrm{f}}\right) \frac{1}{\mathfrak{D}_{A}+i}\right),
$$


and the fact that $A+\boldsymbol{J} A \boldsymbol{J}^{-1}+\Gamma \otimes \mathscr{D}_{\mathrm{f}}$ is bounded, we see that the resolvent of $\mathscr{D}_{A}$ belongs to $\mathscr{L}^{8+\varepsilon}\left(\mathscr{H} \otimes \mathscr{H}_{\mathrm{f}}\right)$ for all $\varepsilon>0$ too. Accordingly, $e^{-t D_{A}^{2}}$ is of trace-class for all $t>0$.

Note also that the bounds of Lemmas 10, 12, 20, Proposition 18 and Corollary 22

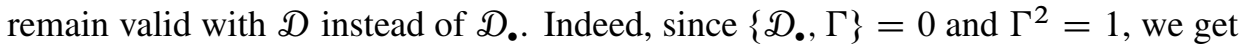
$\mathscr{D}^{2}=\mathscr{D}_{\bullet}^{2} \otimes 1+1 \otimes \mathscr{D}_{\mathrm{f}}^{2}$ and thus

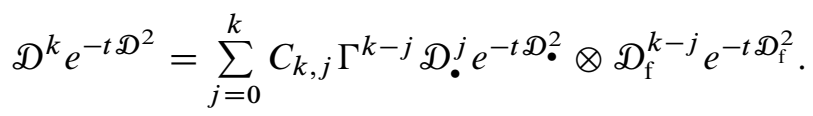

This implies for $f, g \in \mathscr{B}_{\star}, a, b \in \mathcal{A}_{\mathrm{f}}$ and $1 \leq p \leq \infty$,

$$
\begin{aligned}
\| L_{\star}(f) & \otimes a \boldsymbol{J} L_{\star}(g) \otimes b \boldsymbol{J}^{-1} \mathscr{D}^{k} e^{-t \mathscr{D}^{2}} \|_{p} \\
& \leq \sum_{j=0}^{k}\left|C_{k, j}\right|\left\|L_{\star}(f) R_{\star}(\bar{g}) \mathscr{D}_{\bullet}^{j} e^{-t D_{\bullet}^{2}}\right\|_{p}\left\|a J_{\mathrm{f}} b J_{\mathrm{f}}^{-1} \mathscr{D}_{\mathrm{f}}^{k-j} e^{-t} \mathscr{D}_{\mathrm{f}}^{2}\right\|_{p} .
\end{aligned}
$$

Thus, we may assume without loss of generality that there is no finite spectral triple in the picture.

We are going to deduce the expansion from the Duhamel principle:

$$
e^{-t(A+B)}=e^{-t A}-t \int_{0}^{1} e^{-s t(A+B)} B e^{-(1-s) t A} d s .
$$

We write $\mathscr{D}_{A}^{2}=\mathscr{D}^{2}+\widetilde{F}_{0}+\widetilde{F}_{1}$, with $\widetilde{F}_{0}:=F_{0}+\boldsymbol{J} F_{0} \boldsymbol{J}^{-1}+2 A \boldsymbol{J} A \boldsymbol{J}$ and $\widetilde{F}_{1}:=$ $F_{1}+\boldsymbol{J} F_{1} J^{-1}$. The operator $\widetilde{F}_{0}$ is bounded, whereas $\widetilde{F}_{1}$ is unbounded but relatively $\mathscr{D}$-bounded. The Duhamel expansion allows us to write (formally first)

$$
e^{-t \mathscr{D}_{A}^{2}}=\sum_{j=0}^{\infty}(-t)^{j} E_{j}(t),
$$

where $E_{0}(t):=e^{-t D^{2}}$ and, for $j>0$,

$$
E_{j}(t):=\sum_{i_{1}, \ldots, i_{j} \in\{0,1\}} \int_{\Delta_{j}} e^{-s_{0} t D^{2}} \widetilde{F}_{i_{1}} e^{-s_{1} t D^{2}} \ldots \widetilde{F}_{i_{j}} e^{-s_{j} t D^{2}} d^{j} s,
$$

and $\triangle_{j}$ denotes the ordinary $j$-simplex,

$$
\triangle_{j}:=\left\{s \in \mathbb{R}^{j+1} \mid s_{k} \geq 0, \sum_{k=0}^{j} s_{k}=1\right\} .
$$

We first show that the sum (24) converges in the trace norm for small values of $t>0$. We only treat the case $j \geq 1$, the case $j=0$ being covered by Lemma 10. For that we use the Hölder inequality (since $\sum_{k=0}^{j} s_{k}=1$ ):

$$
\begin{array}{r}
\left\|E_{j}(t)\right\|_{1} \leq \sum_{i_{1}, \ldots, i_{j} \in\{0,1\}} \int_{\Delta_{j}}\left\|e^{-s_{0} t \mathfrak{D}^{2}} \widetilde{F}_{i_{1}} e^{-s_{1} t \mathfrak{D}^{2}}\right\|_{\left(s_{0}+s_{1}\right)}-1\left\|\widetilde{F}_{i_{2}} e^{-s_{2} t \mathfrak{D}^{2}}\right\|_{s_{2}^{-1}} \ldots \\
\ldots\left\|\widetilde{F}_{i_{j}} e^{-s_{j} t \mathscr{D}^{2}}\right\|_{s_{j}^{-1}} d^{j} s .
\end{array}
$$


Then we use the estimate of Proposition 18 and Lemma 21 for $k=2, \ldots, j$ (see the Remark 19 for the precise value of the constants):

$$
\left\|\widetilde{F}_{i_{k}} e^{-s_{k} t D^{2}}\right\|_{s_{k}^{-1}} \leq \begin{cases}4\|\tilde{F}\|^{2} C_{1}(\tilde{F})^{s_{k}} t^{-2 s_{k}} & \text { if } i_{k}=0 \\ 2\|\tilde{F}\| C_{2}(\tilde{F})^{s_{k}} t^{-2 s_{k}}\left(t s_{k}\right)^{-\frac{1}{2}} & \text { if } i_{k}=1\end{cases}
$$

For the case $i_{k}=1$, we need to use the factorization property of the algebra of Schwartz functions with Moyal product (as in the proof of Lemma 12), to expand $A$ as a finite sum of products of elements in $\mathcal{A}_{\star} \otimes M_{16}(\mathbb{C})$, and then we can proceed as for the other factors. Taking into account that there are $2^{j}$ such terms and that

$$
\int_{\Delta_{j}} \prod_{i=0}^{j} s_{i}^{-\frac{1}{2}} d^{j} s \leq 2^{j}
$$

we get, since $\sum_{k=0}^{j} s_{k}=1$, the rough estimate

$$
\left\|E_{j}(t)\right\|_{1} \leq 2^{j}\left(4 C_{1}(\tilde{F})\|\widetilde{F}\|^{2}+2 C_{2}(\tilde{F})\|\widetilde{F}\|\right)^{j} t^{-j / 2-2} .
$$

Thus the sum $\sum_{j=0}^{\infty}(-t)^{j} E_{j}(t)$ converges absolutely in the trace-norm for small values of $t$. These estimates also show that

$$
\left|\sum_{j=5}^{\infty}(-t)^{j} \operatorname{Tr}\left(E_{j}(t)\right)\right|=O\left(t^{\frac{1}{2}}\right), \quad t \rightarrow 0,
$$

and accordingly, we only need to consider the terms $(-t)^{j} \operatorname{Tr}\left(E_{j}(t)\right)$ for $j=0,1,2,3$ and 4.

Note first that

$$
\begin{aligned}
\operatorname{Tr}\left(-t E_{1}(t)\right) & =\int_{0}^{1} \operatorname{Tr}\left(-t e^{-s_{0} t \mathscr{D}^{2}}\left(\widetilde{F}_{0}+\widetilde{F}_{1}\right) e^{-\left(1-s_{0}\right) t \mathscr{D}^{2}}\right) d s_{0} \\
& =\operatorname{Tr}\left(-t\left(\widetilde{F}_{0}+\widetilde{F}_{1}\right) e^{-t \mathscr{D}^{2}}\right) .
\end{aligned}
$$

For $j=2,3$ and 4 , we use the relation (15) to collect the heat operators as follows:

$$
\begin{aligned}
& E_{2}(t)=\int_{\Delta_{2}} e^{-s_{0} t \mathscr{D}^{2}}\left(\widetilde{F}_{0}+\widetilde{F}_{1}\right) e^{-s_{1} t \mathscr{D}^{2}}\left(\widetilde{F}_{0}+\widetilde{F}_{1}\right) e^{-s_{2} t D^{2}} d s_{0} d s_{1} d s_{2} \\
& =\int_{0}^{1} d s_{0} \int_{0}^{1-s_{0}} d s_{1} e^{-s_{0} t \mathscr{D}^{2}}\left(\widetilde{F}_{0}+\widetilde{F}_{1}\right) e^{-s_{1} t \mathscr{D}^{2}}\left(\widetilde{F}_{0}+\widetilde{F}_{1}\right) e^{-\left(1-s_{0}-s_{1}\right) t \mathscr{D}^{2}} \\
& =\int_{0}^{1} d s_{0} \int_{0}^{1-s_{0}} d s_{1} e^{-s_{0} t \mathscr{D}^{2}}\left(\widetilde{F}_{0}+\widetilde{F}_{1}\right)^{2} e^{-\left(1-s_{0}\right) t D^{2}}-t \int_{0}^{1} d s_{0} \int_{0}^{1-s_{0}} s_{1} d s_{1} \\
& \cdot \int_{0}^{1} d r_{1} e^{-s_{0} t \mathscr{D}^{2}}\left(\widetilde{F}_{0}+\widetilde{F}_{1}\right) e^{-r_{1} s_{1} t \mathscr{D}^{2}}\left[\mathscr{D}^{2},\left(\widetilde{F}_{0}+\widetilde{F}_{1}\right)\right] e^{-\left(1-s_{0}-r_{1} s_{1}\right) t \mathscr{D}^{2}}
\end{aligned}
$$


Spectral geometry of the Moyal plane with harmonic propagation

$$
\begin{aligned}
& =\int_{0}^{1} d s_{0} \int_{0}^{1-s_{0}} d s_{1} e^{-s_{0} t \mathcal{D}^{2}}\left\{\left(\widetilde{F}_{0}+\widetilde{F}_{1}\right)^{2}-t s_{1}\left(\widetilde{F}_{0}+\widetilde{F}_{1}\right)\left[\mathcal{D}^{2},\left(\widetilde{F}_{0}+\widetilde{F}_{1}\right)\right]\right\} \\
& \cdot e^{-\left(1-s_{0}\right) t \mathscr{D}^{2}}+t^{2} \int_{0}^{1} d s_{0} \int_{0}^{1-s_{0}} s_{1}^{2} d s_{1} \int_{0}^{1} r_{1} d r_{1} \int_{0}^{1} d r_{2} e^{-s_{0} t \mathscr{D}^{2}}\left(\widetilde{F}_{0}+\widetilde{F}_{1}\right) \\
& \cdot e^{-r_{1} r_{2} s_{1} t \mathscr{D}^{2}}\left[\mathscr{D}^{2},\left[\mathscr{D}^{2},\left(\tilde{F}_{0}+\widetilde{F}_{1}\right)\right]\right] e^{-\left(1-s_{0}-r_{1} r_{2} s_{1}\right) t D^{2}} \\
& =\int_{0}^{1} d s_{0} \int_{0}^{1-s_{0}} d s_{1} e^{-s_{0} t \mathscr{D}^{2}}\left\{\left(\widetilde{F}_{0}+\widetilde{F}_{1}\right)^{2}-t s_{1}\left(\widetilde{F}_{0}+\widetilde{F}_{1}\right)\left[\mathscr{D}^{2},\left(\widetilde{F}_{0}+\widetilde{F}_{1}\right)\right]\right. \\
& \left.+\frac{t^{2}}{2} s_{1}^{2}\left(\widetilde{F}_{0}+\widetilde{F}_{1}\right)\left[\mathscr{D}^{2},\left[\mathscr{D}^{2},\left(\widetilde{F}_{0}+\widetilde{F}_{1}\right)\right]\right]\right\} e^{-\left(1-s_{0}\right) t D^{2}} \\
& -t^{3} \int_{0}^{1} d s_{0} \int_{0}^{1-s_{0}} s_{1}^{3} d s_{1} \int_{0}^{1} r_{1}^{2} d r_{1} \int_{0}^{1} r_{2} d r_{2} \int_{0}^{1} d r_{3} e^{-s_{0} t \mathscr{D}^{2}}\left(\widetilde{F}_{0}+\widetilde{F}_{1}\right) \\
& \cdot e^{-r_{1} r_{2} r_{3} s_{1} t \mathscr{D}^{2}}\left[\mathscr{D}^{2},\left[\mathscr{D}^{2},\left[\mathscr{D}^{2},\left(\widetilde{F}_{0}+\widetilde{F}_{1}\right)\right]\right]\right] e^{-\left(1-s_{0}-r_{1} r_{2} r_{3} s_{1}\right) t \mathscr{D}^{2}} .
\end{aligned}
$$

Since the principal symbol of $\mathscr{D}^{2}$ is scalar, we see that $\left[\mathscr{D}^{2},\left[\mathscr{D}^{2},\left[\mathscr{D}^{2}, \widetilde{F}_{1}\right]\right]\right]$ has order 4 in $\nabla_{\mu}^{a}$. Thus, Lemma 20 shows that the last integral (multiplied by its $t^{2}$ global prefactor) gives rise to a trace-class operator with trace of order $t^{\frac{1}{2}}$. Taking the trace and using that $\widetilde{F}_{j}$ is of order $j$ in $\nabla_{\mu}^{a}$, we find up to terms that vanish when $t \rightarrow 0$ :

$$
\begin{aligned}
\operatorname{Tr}\left(t^{2} E_{2}(t)\right)= & \operatorname{Tr}\left(\left\{\frac{t^{2}}{2}\left(\widetilde{F}_{0}+\widetilde{F}_{1}\right)^{2}-\frac{t^{3}}{6}\left(\widetilde{F}_{0}\left[\mathscr{D}^{2}, \widetilde{F}_{1}\right]+\widetilde{F}_{1}\left[\mathscr{D}^{2}, \widetilde{F}_{0}\right]+\widetilde{F}_{1}\left[\mathscr{D}^{2}, \widetilde{F}_{1}\right]\right)\right.\right. \\
& \left.\left.+\frac{t^{4}}{24} \widetilde{F}_{1}\left[\mathscr{D}^{2},\left[\mathscr{D}^{2}, \widetilde{F}_{1}\right]\right]\right\} e^{-t \mathscr{D}^{2}}\right)+\mathcal{O}(\sqrt{t}) .
\end{aligned}
$$

It will be more convenient to write $\operatorname{Tr}\left(F_{1}\left[\mathscr{D}^{2}, F_{0}\right] e^{-t \mathscr{D}^{2}}\right)=\operatorname{Tr}\left(-\left[\mathscr{D}^{2}, F_{1}\right] F_{0} e^{-t \mathscr{D}^{2}}\right)$. By similar arguments one finds

$$
\begin{aligned}
\operatorname{Tr}\left(-t^{3} E_{3}(t)\right)= & \operatorname{Tr}\left(\left\{-\frac{t^{3}}{6}\left(\widetilde{F}_{0} \widetilde{F}_{1}^{2}+\widetilde{F}_{1} \widetilde{F}_{0} \widetilde{F}_{1}+\widetilde{F}_{1}^{2} \widetilde{F}_{0}+\widetilde{F}_{1}^{3}\right)\right.\right. \\
& \left.\left.+\frac{t^{4}}{24}\left(2 \widetilde{F}_{1}^{2}\left[\mathscr{D}^{2}, \widetilde{F}_{1}\right]+\widetilde{F}_{1}\left[\mathscr{D}^{2}, \widetilde{F}_{1}\right] \widetilde{F}_{1}\right)\right\} e^{-t D^{2}}\right)+\mathcal{O}(\sqrt{t}),
\end{aligned}
$$

and lastly

$$
\operatorname{Tr}\left(t^{4} E_{4}(t)\right)=\operatorname{Tr}\left(\frac{t^{4}}{24} \widetilde{F}_{1}^{4} e^{-t \mathfrak{D}^{2}}\right)+\mathcal{O}(\sqrt{t}) .
$$

In summary, we have

$$
\begin{aligned}
\operatorname{Tr}\left(e^{-t D_{A}^{2}}\right)= & \operatorname{Tr}\left(\left\{1-t\left(\widetilde{F}_{0}+\widetilde{F}_{1}\right)+\frac{t^{2}}{2}\left(\widetilde{F}_{0}^{2}+\widetilde{F}_{1} \widetilde{F}_{0}+\widetilde{F}_{0} \widetilde{F}_{1}+\widetilde{F}_{1}^{2}\right)\right.\right. \\
& -\frac{t^{3}}{6}\left(\widetilde{F}_{0}\left[\mathscr{D}^{2}, \widetilde{F}_{1}\right]-\left[\mathscr{D}^{2}, \widetilde{F}_{1}\right] \widetilde{F}_{0}+\widetilde{F}_{1}\left[\mathscr{D}^{2}, \widetilde{F}_{1}\right]+\widetilde{F}_{0} \widetilde{F}_{1}^{2}\right. \\
& \left.+\widetilde{F}_{1} \widetilde{F}_{0} \widetilde{F}_{1}+\widetilde{F}_{1}^{2} \widetilde{F}_{0}+\widetilde{F}_{1}^{3}\right)+\frac{t^{4}}{24}\left(\widetilde{F}_{1}\left[\mathscr{D}^{2},\left[\mathscr{D}^{2}, \widetilde{F}_{1}\right]\right]\right. \\
& \left.\left.\left.+2 \widetilde{F}_{1}^{2}\left[\mathscr{D}^{2}, \widetilde{F}_{1}\right]+\widetilde{F}_{1}\left[\mathscr{D}^{2}, \widetilde{F}_{1}\right] \widetilde{F}_{1}+\widetilde{F}_{1}^{4}\right)\right\} e^{-t \mathscr{D}^{2}}\right)+\mathcal{O}(\sqrt{t}) .
\end{aligned}
$$

Now we can take into account the result of Lemma 21, which says in this context that mixed products $F_{i} \boldsymbol{J} F_{j} \boldsymbol{J}^{-1}$ are already trace-class. Since $J L_{\star}(g) J^{-1}=R_{\star}(\bar{g})$, 
with $R_{\star}$ the right regular representation, we see by Lemma 4 that all terms in (25) with products of $F_{i}$ and $\boldsymbol{J} F_{j} \boldsymbol{J}^{-1}$ are (up to matrices) of the form

$$
L_{\star}(f) R_{\star}(g) \prod_{\mu=1}^{4}\left(\hat{\nabla}_{\mu}\right)^{\alpha_{\mu}} e^{-t H},
$$

with $|\alpha|$ not exceeding the number of $F_{1}$ plus the number of commutators by $\mathscr{D}^{2}$. This argument also relies on the fact that $\boldsymbol{J}$ commutes with $\hat{\nabla}_{\mu}$ according to (13). Thus, Corollary 22 shows that the cross-terms, i.e., the terms with powers of both $F_{i}$ and $\boldsymbol{J} F_{j} \boldsymbol{J}^{-1}$ resulting from products of $\widetilde{F}_{0}=F_{0}+\boldsymbol{J} F_{0} \boldsymbol{J}^{-1}+2 F_{-1} \boldsymbol{J} F_{-1} \boldsymbol{J}^{-1}$ and $\widetilde{F}_{1}=F_{1}+J F_{1} J^{-1}$, where $F_{-1}:=A$, give rise to vanishing contributions in the limit $t \rightarrow 0$. Thus, only the terms with either powers of $F_{i}$ or powers of $\boldsymbol{J} F_{i} \boldsymbol{J}^{-1}$ do contribute to the diverging part of this asymptotic expansion. Since moreover $\boldsymbol{J}$ commutes with $\mathscr{D}$ (we are in even KO-dimension), the trace property shows that both terms (with only $A$ or only $\boldsymbol{J}_{A} \boldsymbol{J}^{-1}$ ) give the same contribution and we get the announced result.

Remark 24. A very important feature of Proposition 23 is that if the heat-trace of the partially fluctuated Dirac operator $\widetilde{D}_{A}:=\mathscr{D}+A, A=\sum_{i} a_{i}\left[\mathscr{D}, b_{i}\right]$, has an asymptotic expansion

$$
\operatorname{Tr}\left(e^{-t \widetilde{\mathfrak{D}}_{A}^{2}}\right)=a_{0} t^{-4}+\sum_{k=1}^{4} a_{k} t^{-2+k / 2}+\mathcal{O}(\sqrt{t}),
$$

then the heat-trace of the fully fluctuated Dirac operator $\mathscr{D}_{A}:=\mathscr{D}+A+\boldsymbol{J} A \boldsymbol{J}^{-1}$ has the asymptotic expansion

$$
\operatorname{Tr}\left(e^{-t D_{A}^{2}}\right)=a_{0} t^{-4}+2 \sum_{k=1}^{4} a_{k} t^{-2+k / 2}+\mathcal{O}(\sqrt{t}),
$$

for the same coefficients $a_{0}, \ldots, a_{k}$. Also, this shows that the asymptotic expansion of the heat-trace of the fully fluctuated Dirac operator is independent of the choice of the real structure $J_{\mathrm{f}}$ of the finite spectral triple $\left(\mathcal{A}_{\mathrm{f}}, \mathscr{H}_{\mathrm{f}}, \mathscr{D}_{\mathrm{f}}\right)$. This fact holds for Moyal spectral triples with harmonic propagation in any (even) dimension.

3.3. Application: the spectral action for the $U\left(\mathscr{B}_{\star}\right)$-Higgs model. In the ConnesLott spirit [10] we take the tensor product of the 4-dimensional spectral triple $\left(\mathcal{A}_{\star}, \mathcal{H}, \mathscr{D}_{\bullet}, \Gamma, J\right), \bullet=1,2$, with the finite Higgs spectral triple $\left(\mathbb{C} \oplus \mathbb{C}, \mathbb{C}^{2}, M \sigma_{1}, J_{\mathrm{f}}\right)$, where $M>0$ and $J_{\mathrm{f}}$ is any real structure. The Dirac operator $\mathscr{D}=\mathscr{D}_{\bullet} \otimes 1+\Gamma \otimes M \sigma_{1}$ of the product triple becomes

$$
\mathscr{D}=\left(\begin{array}{cc}
\mathscr{D} & M \Gamma \\
M \Gamma & \mathscr{D}_{\bullet}
\end{array}\right)
$$


In this representation, the algebra is $\mathcal{A}_{\star} \oplus \mathcal{A}_{\star} \ni(f, g)$, which acts on $\mathscr{H} \oplus \mathscr{H}$ by diagonal left Moyal multiplication. The commutator of $\mathscr{D}$ with $(f, g)$ is, in case that $\mathscr{D}_{1}$ is chosen, according to (9) given by

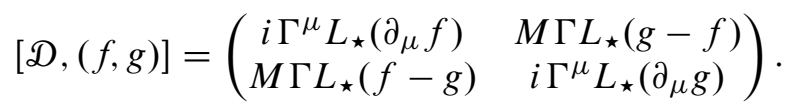

(If we choose $\mathscr{D}_{2}$ instead, then $\Gamma^{\mu}$ has to be replaced by $\Gamma^{\mu+4}$ everywhere.) This shows that the selfadjoint fluctuation $A=\sum_{i} a_{i}\left[\mathcal{D}, b_{i}\right]$ of the fluctuated Dirac operators $\mathscr{D}_{A}=\mathscr{D}+A+\boldsymbol{J} A \boldsymbol{J}^{-1}, \boldsymbol{J}=J \otimes J_{\mathrm{f}}$, is of the form

$$
A=\left(\begin{array}{cc}
\Gamma^{\mu} L_{\star}\left(A_{\mu}\right) & \Gamma L_{\star}(\phi) \\
\Gamma L_{\star}(\bar{\phi}) & \Gamma^{\mu} L_{\star}\left(B_{\mu}\right)
\end{array}\right),
$$

for two real one-forms $A_{\mu}, B_{\mu} \in \mathcal{A}_{\star}$ and a one complex field $\phi \in \mathcal{A}_{\star}$. Again, this holds for $\mathscr{D}_{1}$; for $\mathscr{D}_{2}$ we have to replace $\Gamma^{\mu}$ by $\Gamma^{\mu+4}$.

In terms of the connection introduced in Lemma 4 and using (11) we identify the relevant operators arising in the expansion $\mathscr{D}_{A}^{2}=\mathscr{D}_{\bullet}^{2}+M^{2}+\left(F_{0}+F_{1}\right)+\boldsymbol{J}\left(F_{0}+\right.$ $\left.F_{1}\right) \boldsymbol{J}^{-1}+2 A \boldsymbol{J} A \boldsymbol{J}^{-1}$ of Proposition 23 as follows:

$$
\begin{aligned}
& F_{0}=\left(\begin{array}{cc}
L_{\star}\left(V_{A, \phi}\right) 1+\frac{i}{4}\left[\Gamma^{\mu}, \Gamma^{\nu}\right] L_{\star}\left(F_{\mu \nu}^{A}\right) & i \Gamma^{\mu} \Gamma L_{\star}\left(D_{\mu} \phi\right) \\
i \Gamma^{\mu} \Gamma L_{\star}\left(\overline{D_{\mu} \phi}\right) & L_{\star}\left(V_{B, \phi}\right) 1+\frac{i}{4}\left[\Gamma^{\mu}, \Gamma^{\nu}\right] L_{\star}\left(F_{\mu \nu}^{B}\right)
\end{array}\right), \\
& F_{1}=\left(\begin{array}{cc}
2 i L_{\star}\left(A^{\mu}\right) \nabla_{\mu} & 0 \\
0 & 2 i L_{\star}\left(B^{\mu}\right) \nabla_{\mu}
\end{array}\right),
\end{aligned}
$$

where

$$
\begin{aligned}
V_{A, \phi} & :=\phi \star \bar{\phi}+M(\phi+\bar{\phi})+\left(g^{-1}\right)^{\mu \nu}\left(i \partial_{\mu} A_{v}+A_{\mu} \star A_{v}\right), \\
V_{B, \phi} & :=\bar{\phi} \star \phi+M(\phi+\bar{\phi})+\left(g^{-1}\right)^{\mu \nu}\left(i \partial_{\mu} B_{v}+B_{\mu} \star B_{v}\right), \\
F_{\mu \nu}^{A} & :=\partial_{\mu} A_{\nu}-\partial_{\nu} A_{\mu}-i\left(A_{\mu} \star A_{v}-A_{v} \star A_{\mu}\right), \\
F_{\mu \nu}^{B} & :=\partial_{\mu} B_{v}-\partial_{\nu} B_{\mu}-i\left(B_{\mu} \star B_{v}-B_{v} \star B_{\mu}\right), \\
D_{\mu} \phi & :=\partial_{\mu} \phi-i A_{\mu} \star \phi+i \phi \star B_{\mu}-i M\left(A_{\mu}-B_{\mu}\right) .
\end{aligned}
$$

We have used

$$
\begin{aligned}
\mathscr{D}_{1} \Gamma^{\sigma} L_{\star}\left(A_{\sigma}\right)+L_{\star}\left(A_{\sigma}\right) \mathscr{D}_{1} \Gamma^{\sigma} \\
\quad=-\Gamma^{\sigma}\left[\mathscr{D}_{1}, L_{\star}\left(A_{\sigma}\right)\right]+2 i\left(g^{-1}\right)^{\mu \nu} L_{\star}\left(\partial_{\mu} A_{\nu}\right)+2 i L_{\star}\left(A^{\mu}\right) \nabla_{\mu} .
\end{aligned}
$$

According to the general asymptotic expansion we have obtained in Proposition 23, the only further commutators we need are $\left[\mathcal{D}^{2}, F_{1}\right]$ and $\left[\mathcal{D}^{2},\left[\mathscr{D}^{2}, F_{1}\right]\right]$. Their expression will easily result from the following computation which relies on the relations in Lemma 4:

$$
\begin{aligned}
& {\left[\mathscr{D}^{2}, 2 i L_{\star}\left(A^{\mu}\right) \nabla_{\mu}\right]} \\
& \quad=-2 i L_{\star}\left(\left(g^{-1}\right)^{\rho \sigma} \partial_{\rho} \partial_{\sigma} A^{\mu}\right) \nabla_{\mu}-4 i L_{\star}\left(\partial^{\nu} A^{\mu}\right) \nabla_{\nu} \nabla_{\mu}+4 \widetilde{\Omega}^{2} \Theta_{\mu \nu} L_{\star}\left(A^{\mu}\right) \tilde{\nabla}^{\nu}, \\
& {\left[\mathscr{D}^{2},\left[\mathscr{D}^{2}, 2 i L_{\star}\left(A^{\mu}\right) \nabla_{\mu}\right]=8 i L_{\star}\left(\partial^{\rho} \partial^{\nu} A^{\mu}\right) \nabla_{\rho} \nabla_{\nu} \nabla_{\mu}+\right.\text { lower order terms. }}
\end{aligned}
$$


We have already computed the matrix trace of $e^{-t \widetilde{\Omega} \Sigma}$ in Lemma 10, and the result is $16 \cosh ^{4}(\widetilde{\Omega} t)$. Using

$-i \Sigma=\left(i b_{\mu}-i b_{\mu}^{*}\right)\left(b^{\mu}+b^{\mu *}\right)=g_{\mu \nu}\left(\Gamma^{v}+\frac{\widetilde{\Omega}}{2} \Theta^{\nu \rho} \Gamma_{\rho+4}\right) g^{\mu \sigma}\left(\Gamma_{\sigma+4}+\frac{\widetilde{\Omega}}{2} \Theta_{\sigma \tau} \Gamma^{\tau}\right)$,

the other matrix traces follow from the Clifford algebra:

$$
\begin{aligned}
& \operatorname{tr}_{\mathbb{C}^{16}}\left(\frac{i}{4}\left[\Gamma^{\mu}, \Gamma^{\nu}\right] \cdot e^{-t \tilde{\Omega} \Sigma}\right)=-8 \widetilde{\Omega} \Theta^{\mu v} t+\mathcal{O}\left(t^{2}\right), \\
& \operatorname{tr}_{\mathbb{C}^{16}}\left(\frac{i}{4}\left[\Gamma^{\mu}, \Gamma^{\nu}\right] \cdot \frac{i}{4}\left[\Gamma^{\rho}, \Gamma^{\sigma}\right] \cdot e^{-t \tilde{\Omega} \Sigma}\right)=4\left(g^{-1}\right)^{\mu \rho}\left(g^{-1}\right)^{v \sigma} \\
& \left.-4\left(g^{-1}\right)^{\mu \sigma}\left(g^{-1}\right)^{v \rho}\right)+\mathcal{O}(t), \\
& \operatorname{tr}_{\mathbb{C}^{16}}\left(i \Gamma^{\mu} \Gamma \cdot i \Gamma^{\nu} \Gamma \cdot e^{-t \tilde{\Omega} \Sigma}\right)=16\left(g^{-1}\right)^{\mu \nu}+\mathcal{O}(t) .
\end{aligned}
$$

In terms of the functionals $\mathcal{T}_{\mu_{1} \ldots \mu_{k}}(f):=\operatorname{Tr}_{L^{2}\left(\mathbb{R}^{4}\right)}\left(L_{\star}(f) \nabla_{\mu_{1}} \ldots \nabla_{\mu_{k}} e^{-t H}\right)$ on $\mathcal{A}_{\star}$ introduced and computed in Proposition 6 and the similar functional

$$
\tilde{\mathcal{T}}_{\mu \nu}(f):=\operatorname{Tr}_{L^{2}\left(\mathbb{R}^{4}\right)}\left(L_{\star}(f) \nabla_{\mu} \tilde{\nabla}_{\nu} e^{-t H}\right)
$$

we obtain from Proposition 23 the trace $\operatorname{Tr}\left(e^{-t \mathscr{D}_{A}^{2}}\right)$ as follows:

$$
\begin{aligned}
& \operatorname{Tr}\left(e^{-t D_{A}^{2}}\right)=e^{-t M^{2}}\left\{16 \cosh ^{4}(\widetilde{\Omega} t) \operatorname{Tr}\left(e^{-t H}\right)-2 t \mathcal{T}\left(16 V_{A, \phi}\right)-2 t \mathcal{T}_{\mu}\left(32 i A^{\mu}\right)\right. \\
& +t^{2} \mathcal{T}\left(16 V_{A, \phi} \star V_{A, \phi}+16\left(g^{-1}\right)^{\mu \nu} D_{\mu} \phi \star \overline{D_{\nu} \phi}+8\left(g^{-1}\right)^{\mu \rho}\left(g^{-1}\right)^{\nu \sigma}\right. \\
& \left.\cdot F_{\mu \nu}^{A} F_{\rho \sigma}^{A}+32 i\left(g^{-1}\right)^{\mu \nu} A_{\mu} \star \partial_{\nu} V_{A, \phi}+16 \widetilde{\Omega} \Theta^{\mu \nu} F_{\mu \nu}^{A}\right) \\
& +t^{2} \mathcal{T}_{\mu}\left(32 i A^{\mu} \star V_{A, \phi}+32 i V_{A, \phi} \star A^{\mu}-64\left(g^{-1}\right)^{v \rho} A^{v} \star \partial_{\rho} A^{\mu}\right) \\
& +t^{2} \mathcal{T}_{\mu v}\left(-64 A^{\mu} \star A^{v}\right)-\frac{t^{3}}{3} \mathcal{T}_{\mu \nu}\left(-4 i\left(\left(V_{A, \phi} \star \partial^{\mu} A^{v}-\partial^{\mu} A^{v} \star V_{A, \phi}\right)\right.\right. \\
& +64 A^{\mu}\left(g^{-1}\right)^{\rho \sigma} \partial_{\rho} \partial_{\sigma} A^{v}+128 A_{\rho}\left(g^{-1}\right)^{\rho \sigma} \partial_{\sigma} \partial^{\mu} A^{v} \\
& -64\left(V_{A, \phi} \star A^{\mu} \star A^{v}+A^{\mu} \star V_{A, \phi} \star A^{v}+A^{\mu} \star A^{v} \star V_{A, \phi}\right) \\
& -128 i\left(g^{-1}\right)^{\rho \sigma}\left(A_{\rho} \star\left(\partial_{\sigma} A^{\mu}\right) \star A^{v}+A_{\rho} \star A^{\mu} \star\left(\partial_{\sigma} A^{v}\right)\right. \\
& \left.\left.+A^{\mu} \star A_{\rho} \star\left(\partial_{\sigma} A^{v}\right)\right)\right)-\frac{t^{3}}{3} \mathcal{T}_{\mu \nu \rho}\left(128 A^{\mu} \star \partial^{v} A^{\rho}\right. \\
& \left.-128 i A^{\mu} \star A^{v} \star A^{\rho}\right)-\frac{t^{3}}{3} \tilde{\mathcal{T}}_{\mu \nu}\left(128 i \widetilde{\Omega}^{2} \Theta^{\rho v} A^{\mu} \star A_{\rho}\right) \\
& +\frac{t^{4}}{12} \mathcal{T}_{\mu \nu \rho \sigma}\left(-256 A^{\mu} \star \partial^{\nu} \partial^{\rho} A^{\sigma}+512 i A^{\mu} \star A^{v} \star \partial^{\rho} A^{\sigma}\right. \\
& \left.\left.+256 i A^{\mu} \star\left(\partial^{v} A^{\rho}\right) \star A^{\sigma}+256 A^{\mu} \star A^{v} \star A^{\rho} \star A^{\sigma}\right)\right\} \\
& +\left\{A_{\mu} \mapsto B_{\mu}, F_{\mu \nu}^{A} \mapsto F_{\mu \nu}^{B}, V_{A, \phi} \mapsto V_{B, \phi}, D_{\mu} \phi \leftrightarrow \overline{D_{\mu} \phi}\right\} \\
& +\mathcal{O}(\sqrt{t}) \text {. }
\end{aligned}
$$

The relevant traces have been computed in Proposition 6. A similar procedure gives $\tilde{\mathcal{T}}_{\mu \nu}(f)=\left(\frac{\tilde{\Omega}}{2 \pi \sinh (2 \widetilde{\Omega} t)}\right)^{2} \int_{\mathbb{R}^{4}} d z \sqrt{\operatorname{det} g} f(z)\left(\tilde{\mathcal{N}}_{\mu \nu}+Z_{\mu} \tilde{Z}_{\nu}\right) e^{-\tilde{\Omega} \tanh (\tilde{\Omega} t)\langle z, g z\rangle}$, 
with $\tilde{Z}_{v}:=-2 i\left(\Theta^{-1} z\right)_{\nu}-2 \tilde{\Omega} \tanh (\widetilde{\Omega} t)(g z)_{\nu}$ and $\tilde{\mathcal{N}}_{\mu \nu}:=2 i\left(\Theta^{-1} g^{-1}\right)_{\mu \nu}+$ $i \widetilde{\Omega}^{2}(g \Theta)_{\mu \nu}-\tilde{\Omega} \tanh (\tilde{\Omega} t) \delta_{\mu \nu}$. This shows that the contribution of $\widetilde{\mathcal{J}}_{\mu \nu}(f)$ is suppressed with $\mathcal{O}(t)$. Inserting these traces into (26) we arrive at

$$
\begin{aligned}
\operatorname{Tr}\left(e^{-t D_{A}^{2}}\right)= & \frac{2}{\widetilde{\Omega}^{4}} t^{-4}-\frac{2 M^{2}}{\widetilde{\Omega}^{4}} t^{-3}+\left(\frac{M^{4}}{\widetilde{\Omega}^{4}}+\frac{8}{3 \widetilde{\Omega}^{2}}\right) t^{-2}-\left(\frac{M^{6}}{3 \widetilde{\Omega}^{4}}+\frac{8 M^{2}}{3 \widetilde{\Omega}^{2}}\right) t^{-1} \\
& +\left(\frac{52}{45}+\frac{M^{8}}{12 \widetilde{\Omega}^{4}}+\frac{4 M^{4}}{3 \widetilde{\Omega}^{2}}\right)-t^{-1} \frac{2-2 M^{2} t}{\pi^{2}} \\
& \int d^{4} x \sqrt{\operatorname{det} g}\left\{\phi \star \bar{\phi}+M(\phi+\bar{\phi})+\widetilde{\Omega}^{2}\left(\left\langle X_{A}, g X_{A}\right\rangle_{\star}-\langle x, g x\rangle_{\star}\right)\right. \\
& \left.+\bar{\phi} \star \phi+M(\phi+\bar{\phi})+\widetilde{\Omega}^{2}\left(\left\langle X_{B}, g X_{B}\right\rangle_{\star}-\langle x, g x\rangle_{\star}\right)\right\} \\
& +\frac{1}{\pi^{2}} \int d^{4} x \sqrt{\operatorname{det} g}\left\{2\left(g^{-1}\right)^{\mu \nu} D_{\mu} \phi \star \overline{D_{\nu} \phi}\right. \\
& +\left(\phi \star \bar{\phi}+M(\phi+\bar{\phi})+\widetilde{\Omega}^{2}\left\langle X_{A}, g X_{A}\right\rangle_{\star}\right)^{2}-\left(\widetilde{\Omega}^{2}\langle x, g x\rangle_{\star}\right)^{2} \\
& +\left(\bar{\phi} \star \phi+M(\phi+\bar{\phi})+\widetilde{\Omega}^{2}\left\langle X_{B}, g X_{B}\right\rangle_{\star}\right)^{2}-\left(\widetilde{\Omega}^{2}\langle x, g x\rangle_{\star}\right)^{2} \\
& +\left(\frac{1}{2}\left(g^{-1}\right)^{\mu \rho}\left(g^{-1}\right)^{\nu \sigma}-\frac{1}{6}\left(g^{-1}+\widetilde{\Omega}^{2} \Theta g \Theta\right)^{\mu \rho}\left(g^{-1}+\widetilde{\Omega}^{2} \Theta g \Theta\right)^{\nu \sigma}\right) \\
& \left.\cdot\left(F_{\mu \nu}^{A} \star F_{\rho \sigma}^{A}+F_{\mu \nu}^{B} \star F_{\rho \sigma}^{B}\right)\right\}+\mathcal{O}(\sqrt{t}),
\end{aligned}
$$

where

$$
X_{A}^{\mu}(x):=x^{\mu}+\Theta^{\mu v} A_{\nu}, \quad\langle X, g Y\rangle_{\star}:=g_{\mu \nu} X^{\mu} \star Y^{v} .
$$

To reduce (26) to (27) we have used

- the traciality (4) of the Moyal product and the resulting cyclicity under the integral,

- integration by parts where appropriate,

$-x^{\mu} \star f=\frac{1}{2}\left\{x^{\mu}, f\right\}_{\star}+\frac{1}{2}\left[x^{\mu}, f\right]_{\star}=\frac{1}{2}\left\{x^{\mu}, f\right\}_{\star}+i \Theta^{\mu \nu} \partial_{\nu} f$ where appropriate,

- symmetries and antisymmetries in the indices.

The matrix $g^{-1}+\widetilde{\Omega}^{2} \Theta g \Theta$ appearing in front of the curvature term in (27) can be equivalently written as

$$
g^{-1}+\widetilde{\Omega}^{2} \Theta g \Theta=g^{-1}+4 g\left(1-g^{-1}\right)=g^{-1}(1-2 g)^{2} .
$$

Since $g^{-1} \geq 1$ according to (10), hence $0 \leq g \leq 1$, we have $0 \leq g^{-1}+\widetilde{\Omega}^{2} \Theta g \Theta \leq$ $g^{-1}$, showing that the matrix in front of the curvature term in (27) is strictly positive. It is minimal for $g=\frac{1}{2}$, i.e., $\widetilde{\Omega}^{2}\left(\Theta^{t} \Theta\right)_{\mu \nu}=4 \delta_{\mu \nu}$. The curvature $F_{\mu \nu}$ can also be expressed in terms of the covariant coordinates, $\left[X_{A}^{\mu}, X_{A}^{\nu}\right]_{\star}=i \Theta^{\mu \nu}+i \Theta^{\mu \rho} \Theta^{\nu \sigma} F_{\rho \sigma}^{A}$.

With the moments $\chi_{-n}=\int_{0}^{\infty} d s s^{n-1} \chi(s)$ of the "characteristic function" and 
$\chi_{0}=\chi(0)$, we identify the spectral action (23) as

$$
\begin{aligned}
S_{\Lambda}\left(D_{A}\right)= & \frac{2 \Lambda^{8}}{\widetilde{\Omega}^{4}} \chi_{-4}-\frac{2 M^{2} \Lambda^{6}}{\widetilde{\Omega}^{4}} \chi_{-3}+\left(\frac{M^{4} \Lambda^{4}}{\widetilde{\Omega}^{4}}+\frac{8 \Lambda^{4}}{3 \widetilde{\Omega}^{2}}\right) \chi_{-2} \\
& -\left(\frac{M^{6} \Lambda^{2}}{3 \widetilde{\Omega}^{4}}+\frac{8 M^{2} \Lambda^{2}}{3 \widetilde{\Omega}^{2}}\right) \chi_{-1}+\left(\frac{52}{45}+\frac{M^{8}}{12 \widetilde{\Omega}^{4}}+\frac{4 M^{4}}{3 \widetilde{\Omega}^{2}}\right) \chi_{0} \\
& +\frac{\chi_{0}}{\pi^{2}} \int d^{4} x \sqrt{\operatorname{det} g}\left\{2\left(g^{-1}\right)^{\mu \nu} D_{\mu} \phi \star \overline{D_{\nu} \phi}+(\phi \star \bar{\phi}+M(\phi+\bar{\phi})\right. \\
& \left.+\widetilde{\Omega}^{2}\left\langle X_{A}, g X_{A}\right\rangle_{\star}+M^{2}-\frac{\chi-1}{\chi_{0}} \Lambda^{2}\right)^{2}-\left(\widetilde{\Omega}^{2}\langle x, g x\rangle_{\star}+M^{2}-\frac{\chi_{-1}}{\chi_{0}} \Lambda^{2}\right)^{2} \\
& +\left(\bar{\phi} \star \phi+M(\phi+\bar{\phi})+\widetilde{\Omega}^{2}\left\langle X_{B}, g X_{B}\right\rangle_{\star}+M^{2}-\frac{\chi_{-1}}{\chi_{0}} \Lambda^{2}\right)^{2} \\
& -\left(\widetilde{\Omega}^{2}\langle x, g x\rangle_{\star}+M^{2}-\frac{\chi-1}{\chi_{0}} \Lambda^{2}\right)^{2}+\left(\frac{1}{2}\left(g^{-1}\right)^{\mu \rho}\left(g^{-1}\right)^{v \sigma}\right. \\
& \left.-\frac{1}{6}\left(g^{-1}+\widetilde{\Omega}^{2} \Theta g \Theta\right)^{\mu \rho}\left(g^{-1}+\widetilde{\Omega}^{2} \Theta g \Theta\right)^{\nu \sigma}\right) \\
& \left.\cdot\left(F_{\mu \nu}^{A} \star F_{\rho \sigma}^{A}+F_{\mu \nu}^{B} \star F_{\rho \sigma}^{B}\right)\right\}+\mathcal{O}\left(\Lambda^{-1}\right) .
\end{aligned}
$$

The final result (28) for the spectral action agrees, up to typos, with the result obtained in [27]. We recall that with the cumbersome computational method of [27] it was only possible to identify the part of the spectral action at most bilinear in the gauge fields $A, B$. By gauge-invariant completion it was argued that the total spectral action has to be (28). In [27] the $\Theta$-matrix was chosen as $\Theta=\theta\left(\begin{array}{cc}i \sigma_{2} & 0 \\ 0 & i \sigma_{2}\end{array}\right)$. In terms of $\Omega:=\frac{\theta \widetilde{\Omega}}{2}$ this choice leads to $\left(g^{-1}\right)^{\mu \nu}=\left(1+\Omega^{2}\right) \delta^{\mu \nu}$ and $\sqrt{\operatorname{det} g}=\frac{1}{\left(1+\Omega^{2}\right)^{2}}$. Up to the global factor of 2 due to the real structure, the few differences in the prefactors ${ }^{2}$ are easily identified as typos in [27]. We finish by a brief discussion of the spectral action:

- The square of covariant coordinates $X_{A}, X_{B}$ combines with the Higgs field $\phi$ to a non-trivial potential. This was not noticed in [13], [21]. We observe here a much deeper unification of the continuous geometry described by Yang-Mills fields and discrete geometry described by the Higgs field than previously in almost-commutative geometry.

- The coefficient in front of the Yang-Mills action is strictly positive for any realvalued $\widetilde{\Omega}$. In the bosonic model of [13], [21] there was only the analogue of the negative part, which leads to problems with the field equations.

Unlike the scalar model renormalized in [24] where $\Omega=\frac{\theta \widetilde{\Omega}}{2}$ can by LangmannSzabo duality be restricted to $\Omega \in[0,1]$, the full spectral action (28) does not have a distinguished frequency parameter $\widetilde{\Omega}>0$.

- The action (28) is invariant under gauge transformations

$$
\begin{gathered}
\phi+M \mapsto u_{A} \star(\phi+M) \star \overline{u_{B}}, \quad X_{A \mu} \mapsto u_{A} \star X_{A \mu} \star \overline{u_{A}}, \\
X_{B \mu} \mapsto u_{B} \star X_{B \mu} \star \overline{u_{B}},
\end{gathered}
$$

${ }^{2}$ These are $\left(\frac{\left(1+\Omega^{2}\right)^{2}}{2}-\frac{\left(1-\Omega^{2}\right)^{4}}{6\left(1+\Omega^{2}\right)^{2}}\right)$ versus $\left(\frac{\left(1-\Omega^{2}\right)^{2}}{2}-\frac{\left(1-\Omega^{2}\right)^{4}}{3\left(1+\Omega^{2}\right)^{2}}\right)$ in [27] in front of $F_{\mu \nu} F^{\mu \nu}$ and $\frac{\left(1+\Omega^{2}\right)}{2}$ versus $\frac{1}{2}$ in [27] in front of $(D \phi)^{2}$. 
where $u_{A}, u_{B} \in U\left(\mathscr{B}_{\star}\right)$ are unital elements of the preferred unitization.

- For any value of the free parameter $\frac{M^{2} \chi_{0}}{\Lambda^{2} \chi_{-1}}$, the action contains $(A, B, \phi)$-linear terms which lead to a complicated vacuum which is not attained at vanishing $A, B, \phi$. Since $A, B, \phi$ are Schwartz functions, the formal vacuum solution $X_{A}=0=X_{B}$ and $\phi+M=\sqrt{\frac{\chi-1}{\chi_{0}}} \Lambda$ is excluded. An enlargement of Schwartz class function to e.g. polynomially bounded functions does not help either, because then we are not allowed to expand the Gaußian $e^{-\widetilde{\Omega} \tanh (\widetilde{\Omega} t)\langle x, g x\rangle_{\star}}$ in $t$, making the spectral action different from (28).

- If we formally regard $\phi+M, X_{A}, X_{B}$ as dynamical variables of the model, then (28) can be viewed as translation-invariant with respect to

$$
\phi(x)+M \mapsto \phi(x+a)+M, \quad X_{A}(x) \mapsto X_{A}(x+a), \quad X_{B}(x) \mapsto X_{B}(x+a) .
$$

This would clear away a frequent objection against the renormalizable $\phi_{4}^{4}$ models, breaking of translation invariance. However, this transformation abandons the space of Schwartz class functions for $A, B, \phi$, so that translation invariance remains broken in the consistent spectral action.

- The vacuum part of the spectral action is finite. In general, the heat kernel expansion for non-compact spectral triples is ill-defined, so that a spatial regularization of the operator trace is unavoidable. See e.g. [19]. The oscillator potential is one of many possibilities. We want to advertise the point of view that if one takes the spectral action principle serious, the spatial regularization is part of the geometry. The removal of the spatial regularization must be carefully studied. In general, we should expect that other limiting procedures such as those of quantum field theory make it impossible to remove the regularization (UV/IR).

\section{Appendix: Locally compact noncommutative spin manifolds}

Definition 25. A non-compact spectral triple is given by the data

$$
(\mathcal{A}, \mathscr{B}, \mathscr{H}, \mathcal{D}, J, \Gamma, c)
$$

satisfying conditions (0)-(6) given below. The data consist of a non-unital algebra $\mathcal{A}$ acting faithfully (via a representation denoted by $\pi$ ) by bounded operators on the Hilbert space $\mathscr{H}$; a preferred unitization $\mathscr{B}$ of $\mathcal{A}$ acting by bounded operators on the same Hilbert space; and an essentially self-adjoint unbounded operator $\mathscr{D}$ on $\mathscr{H}$ such that $[\mathcal{D}, \pi(a)]$ extends to a bounded operator for any $a \in \mathscr{B}$. The spectral triple is said to be even if there exists a $\mathbb{Z}_{2}$-grading operator $\Gamma$ on $\mathcal{H}$ satisfying $\Gamma^{2}=1$, for which $\mathscr{B}$ is even and $\mathscr{D}$ is odd. The spectral triple is said to be real if there exists an antiunitary operator $J$ on $\mathscr{H}$ which satisfies conditions (4) and (5) below. 
(0) Compactness. The operator $\pi(a)(\mathscr{D}-\lambda)^{-1}$ is compact for all $a \in \mathcal{A}$ and $\lambda$ in the resolvent set of $\mathscr{D}$.

(1) Regularity. For any $a \in \mathscr{B}$, both $\pi(a)$ and $[\mathscr{D}, \pi(a)]$ belong to $\bigcap_{n=1}^{\infty} \operatorname{dom}\left(\delta^{n}\right)$, with $\delta(T):=[\langle\mathscr{D}\rangle, T]$ and $\langle\mathscr{D}\rangle:=\left(\mathscr{D}^{2}+1\right)^{\frac{1}{2}}$.

For any element $b$ of the algebra $\Psi_{0}(\mathcal{A})$ generated by $\delta^{n}(\pi(\mathcal{A}))$ and

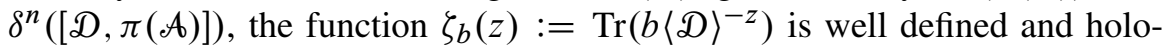
morphic for $\operatorname{Re}(z)$ large and analytically continues to $\mathbb{C} \backslash \operatorname{Sd}$ for some discrete set $\mathrm{Sd} \subset \mathbb{C}$ (the dimension spectrum). Moreover the dimension spectrum is said to be simple if all the poles are simple, finite if there is $k \in \mathbb{N}$ such that all the poles are order at most $k$ and if not, infinite.

(2) Metric dimension. For the metric dimension $d:=\sup \{\operatorname{Re}(z), z \in \mathrm{Sd}\}$, the operator $\pi(a)\langle\mathscr{D}\rangle^{-d}$ belongs to the Dixmier ideal $\mathscr{L}^{1, \infty}(\mathscr{H})$ for any $a \in \mathcal{A}$. Moreover, for any Dixmier trace, the map $\mathcal{A}_{+} \ni a \mapsto \operatorname{Tr}_{\omega}\left(\pi(a)\langle\mathscr{D}\rangle^{-d}\right)$ is non-vanishing.

(3) Finiteness. ${ }^{3}$ The algebra $\mathcal{A}$ and its preferred unitization $\mathscr{B}$ are pre- $C^{\star}$-algebras, i.e., each one is a $\star$-subalgebra of some $C^{*}$-algebra and stable under holomorphic functional calculus.

The space of smooth spinors $\mathscr{H}^{\infty}:=\bigcap_{k=0}^{\infty} \mathscr{H}^{k}$, with $\mathscr{H}^{k}:=\operatorname{dom}\left(D^{k}\right)$ completed with norm $\|\xi\|_{k}^{2}:=\|\xi\|^{2}+\left\|D^{k} \xi\right\|^{2}$, is a finitely generated projective $\mathcal{A}$-module $p \mathcal{A}^{m}$, for some $m \in \mathbb{N}$ and some projector $p=p^{2}=p^{*} \in M_{m}(\mathscr{B})$. The composition of the Dixmier trace with the induced hermitian structure

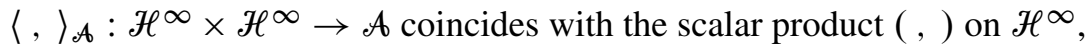

$$
(\xi, \eta)=\operatorname{Tr}_{\omega}\left(\langle\xi, \eta\rangle_{\mathcal{A}}\langle\mathscr{D}\rangle^{-d}\right), \quad \xi, \eta \in \mathscr{H}^{\infty} .
$$

(4) Reality. The operator $J$ defines a real structure of KO-dimension $k \in \mathbb{Z}_{8}$. This means

$$
J^{2}=\varepsilon, \quad J \mathscr{D}=\varepsilon^{\prime} \mathscr{D} J, \quad J \Gamma=\varepsilon^{\prime \prime} \Gamma J \quad \text { (even case) }
$$

with signs $\varepsilon, \varepsilon^{\prime}, \varepsilon^{\prime \prime} \in\{-1,1\}$ given as a function of $k \bmod 8$ by

\begin{tabular}{|c|rrrrrrrr|}
\hline$k$ & 0 & 1 & 2 & 3 & 4 & 5 & 6 & 7 \\
\hline \hline$\varepsilon$ & 1 & 1 & -1 & -1 & -1 & -1 & 1 & 1 \\
$\varepsilon^{\prime}$ & 1 & -1 & 1 & 1 & 1 & -1 & 1 & 1 \\
$\varepsilon^{\prime \prime}$ & 1 & & -1 & & 1 & & -1 & \\
\hline
\end{tabular}

Additionally, the action $\pi$ of $\mathscr{B}$ on $\mathscr{H}$ satisfies the commutation rule $\left[\pi(f), \pi^{o}(g)\right]=0$ for all $f, g \in \mathscr{B}$, where $\pi^{o}(g)=J \pi\left(g^{*}\right) J^{-1}$ is the action of the opposite algebra $\mathscr{B}^{o}$.

\footnotetext{
${ }^{3}$ This is a simplified version of those in [18], as in there the authors are forced to use a third algebra which possesses quasi-local units.
} 
(5) First order. $\left[[\mathcal{D}, \pi(f)], \pi^{o}(g)\right]=0$ for all $f, g \in \mathscr{B}$.

(6) Orientability. ${ }^{4}$ Whenever the metric dimension $d$ is an integer, there is a Hochschild $d$-cycle $c$ on $\mathscr{B}$ with values in $\mathscr{B} \otimes \mathscr{B}^{o}$, i.e., a finite sum of terms $\left(a_{0} \otimes b_{0}\right) \otimes a_{1} \otimes \cdots \otimes a_{d}$. Its representation $\pi_{\mathscr{D}}(c)$ with $\pi_{\mathscr{D}}\left(\left(a_{0} \otimes b_{0}\right) \otimes a_{1} \otimes\right.$ $\left.\cdots \otimes a_{d}\right):=\pi\left(a_{0}\right) J \pi\left(b_{0}^{*}\right) J^{-1}\left[\mathcal{D}, \pi\left(a_{1}\right)\right] \ldots\left[\mathcal{D}, \pi\left(a_{d}\right)\right]$ satisfies $\pi_{\mathscr{D}}(c)^{2}=1$ and defines the volume form on $\mathcal{A}$, i.e.,

$$
\phi_{c}\left(f_{0}, \ldots, f_{d}\right)=\operatorname{Tr}_{\omega}\left(\pi_{\mathscr{D}}(\boldsymbol{c}) \pi\left(f_{0}\right)\left[\mathscr{D}, \pi\left(f_{1}\right)\right] \ldots\left[\mathscr{D}, \pi\left(f_{d}\right)\right]\langle\mathscr{D}\rangle^{-d}\right)
$$

provides a non-vanishing Hochschild $d$-cocycle $\phi_{c}$ on $\mathcal{A}$.

Acknowledgments. The second author would like to thank Harald Grosse for the long-term collaboration which initiated this paper through the preprint [27]. Both authors would like to thank Alan Carey for stimulating discussions and the referee for enlightening comments.

\section{References}

[1] R. Beals, Characterization of pseudodifferential operators and applications. Duke Math. J. 44 (1977), 45-57. Zbl 0353.35088 MR 0435933

[2] A.-P. Calderón and R. Vaillancourt, On the boundedness of pseudo-differential operators. J. Math. Soc. Japan 23 (1971), 374-378. Zbl 0203.45903 MR 0284872

[3] A. Carey, V. Gayral, A. Rennie and F. Sukochev, Index theory for locally compact noncommutative geometries. Mem. Amer. Math. Soc., to appear; preprint 2011. arXiv:1107.0805

[4] A. L. Carey, V. Gayral, A. Rennie, and F. A. Sukochev, Integration on locally compact noncommutative spaces. J. Funct. Anal. 263 (2012), 383-414. Zbl 1251.46036 MR 2923417

[5] A. H. Chamseddine and A. Connes, The spectral action principle. Comm. Math. Phys. 186 (1997), 731-750. Zbl 0894.58007 MR 1463819

[6] A. H. Chamseddine and A. Connes, Scale invariance in the spectral action. J. Math. Phys. 47 (2006), 063504, 19. Zbl 1112.83036 MR 2239979

[7] A. Connes, Gravity coupled with matter and the foundation of non-commutative geometry. Comm. Math. Phys. 182 (1996), 155-176. Zbl 0881.58009 MR 1441908

[8] A. Connes, Noncommutative geometry and the standard model with neutrino mixing. $J$. High Energy Phys. 2006 (2006), 081. MR 2270385

[9] A. Connes, On the spectral characterization of manifolds. J. Noncommut. Geom. 7 (2013), 1-82. Zbl 06155603 MR 3032810

[10] A. Connes and J. Lott, Particle models and noncommutative geometry. Nuclear Phys. B Proc. Suppl. 18B (1990), 29-47. Zbl 0957.46516 MR 1128127

\footnotetext{
${ }^{4}$ In the standard formulation of the orientability condition, and in the case of an even spectral dimension, $\pi_{\mathscr{D}}(c)=\Gamma$. We didn't include this extra condition here as it fails in our example, see (12).
} 
[11] H. O. Cordes, On pseudo-differential operators and smoothness of special Lie-group representations. Manuscripta Math. 28 (1979), 51-69. Zbl 0415.35083 MR 535694

[12] L. Dạbrowski and A. Sitarz, Dirac operator on the standard Podleś quantum sphere. In Noncommutative geometry and quantum groups (Warsaw, 2001), Banach Center Publ. 61, Polish Acad. Sci., Warsaw 2003, 49-58. Zbl 1061.58004 MR 2024421

[13] A. de Goursac, J.-C. Wallet, and R. Wulkenhaar, Noncommutative induced gauge theory. Eur. Phys. J. C Part. Fields 51 (2007), 977-987. Zbl 1189.81215 MR 2336186

[14] M. Disertori, R. Gurau, J. Magnen and V. Rivasseau, Vanishing of beta function of noncommutative $\phi_{4}^{4}$ theory to all orders. Phys. Lett. B 649 (2007), 95-102. Zbl 1248.81253 MR 2308801

[15] M. Disertori and V. Rivasseau, Two- and three-loop beta function of non-commutative $\phi_{4}^{4}$ theory. Eur. Phys. J. C Part. Fields 50 (2007), 661-671. Zbl 1248.81254

[16] J. Dixmier and P. Malliavin, Factorisations de fonctions et de vecteurs indéfiniment différentiables. Bull. Sci. Math. (2) 102 (1978), 305-330. Zbl 0392.43013 MR 517765

[17] D. Essouabri, B. Iochum, C. Levy, and A. Sitarz, Spectral action on noncommutative torus. J. Noncommut. Geom. 2 (2008), 53-123. Zbl 1156.46046 MR 2366124

[18] V. Gayral, J. M. Gracia-Bondía, B. Iochum, T. Schücker, and J. C. Várilly, Moyal planes are spectral triples. Comm. Math. Phys. 246 (2004), 569-623. Zbl 1084.58008 MR 2053945

[19] V. Gayral and B. Iochum, The spectral action for Moyal phase. J. Math. Phys. 46 (2005), 043503. Zbl 1067.58020 MR 2131255

[20] J. M. Gracia-Bondía and J. C. Várilly, Algebras of distributions suitable for phase-space quantum mechanics. I. J. Math. Phys. 29 (1988), 869-879. Zbl 04063802 MR 940351

[21] H. Grosse and M. Wohlgenannt, Induced gauge theory on a noncommutative space. Eur. Phys. J. C Part. Fields 52 (2007), 435-450. Zbl 1189.81217 MR 2346157

[22] H. Grosse and R. Wulkenhaar, The $\beta$-function in duality-covariant non-commutative $\phi^{4}$ theory. Eur. Phys. J. C Part. Fields 35 (2004), 277-282. Zbl 1191.81207 MR 2075076

[23] H. Grosse and R. Wulkenhaar, Power-counting theorem for non-local matrix models and renormalisation. Comm. Math. Phys. 254 (2005), 91-127. Zbl 1079.81049 MR 2116739

[24] H. Grosse and R. Wulkenhaar, Renormalisation of $\phi^{4}$-theory on noncommutative $\mathbb{R}^{4}$ in the matrix base. Comm. Math. Phys. 256 (2005), 305-374. Zbl 1075.82005 MR 2160797

[25] H. Grosse and R. Wulkenhaar, Renormalisation of $\phi^{4}$-theory on non-commutative $\mathbb{R}^{4}$ to all orders. Lett. Math. Phys. 71 (2005), 13-26. Zbl 1115.81055 MR 2136734

[26] H. Grosse and R. Wulkenhaar, Progress in solving a noncommutative quantum field theory in four dimensions. Preprint 2009. arXiv:0909.1389

[27] H. Grosse and R. Wulkenhaar, 8D-spectral triple on 4D-Moyal space and the vacuum of noncommutative gauge theory. J. Geom. Phys. 62 (2012), 1583-1599. Zbl 1243.58005 MR 2922023

[28] R. Gurau, J. Magnen, V. Rivasseau, and F. Vignes-Tourneret, Renormalization of noncommutative $\phi_{4}^{4}$ field theory in $x$ space. Comm. Math. Phys. 267 (2006), 515-542. Zbl 1113.81101 MR 2249779

[29] R. Gurau and V. Rivasseau, Parametric representation of noncommutative field theory. Comm. Math. Phys. 272 (2007), 811-835. Zbl 1156.81465 MR 2304476 
[30] J. Magnen and V. Rivasseau, Constructive $\phi^{4}$ field theory without tears. Ann. Henri Poincaré 9 (2008), 403-424. Zbl 1141.81022 MR 2399194

[31] A. Rennie, Smoothness and locality for nonunital spectral triples. K-Theory 28 (2003), 127-165. Zbl 1027.46088 MR 1995874

[32] V. Rivasseau, Non-commutative renormalization. In Quantum spaces, Prog. Math. Phys. 53, Birkhäuser, Basel 2007, 19-107. Zbl 1139.81047 MR 2382235

[33] V. Rivasseau, Constructive matrix theory. J. High Energy Phys. 2007 (2007), 008. MR 2342423

[34] V. Rivasseau, F. Vignes-Tourneret, and R. Wulkenhaar, Renormalisation of noncommutative $\phi^{4}$-theory by multi-scale analysis. Comm. Math. Phys. 262 (2006), 565-594. Zbl 1109.81056 MR 2202303

[35] B. Simon, Trace ideals and their applications. London Math. Soc. Lecture Note Ser. 35, Cambridge University Press, Cambridge 1979. Zbl 0423.47001 MR 541149

[36] R. Wulkenhaar, Non-compact spectral triples with finite volume. In Quanta of maths, Clay Math. Proc. 11, Amer. Math. Soc., Providence, RI, 2010, 617-648. Zbl 1222.58023 MR 2732067

Received August 26, 2011

V. Gayral, Laboratoire de Mathématiques, U.F.R. Sciences Exactes et Naturelles Moulin de la Housse - BP 1039, 51687 Reims Cedex 2, and Laboratoire de Mathématiques et d'Applications de Metz, ISGMP, Bâtiment A, Île du Saulcy, 57045 Metz Cedex 1, France E-mail: victor.gayral@univ-reims.fr

R. Wulkenhaar, Mathematisches Institut der Westfälischen Wilhelms-Universität Münster, Einsteinstraße 62, 48149 Münster, Germany

E-mail: raimar@math.uni-muenster.de 